\title{
TreeFuser: A Framework for Analyzing and Fusing General Recursive Tree Traversals
}

\author{
LAITH SAKKA, Purdue University, USA \\ KIRSHANTHAN SUNDARARAJAH, Purdue University, USA \\ MILIND KULKARNI, Purdue University, USA
}

Series of traversals of tree structures arise in numerous contexts: abstract syntax tree traversals in compiler passes, rendering traversals of the DOM in web browsers, kd-tree traversals in computational simulation codes. In each of these settings, a tree is traversed multiple times to compute various values and modify various portions of the tree. While it is relatively easy to write these traversals as separate small updates to the tree, for efficiency reasons, traversals are often manually fused to reduce the number of times that each portion of the tree is traversed: by performing multiple operations on the tree simultaneously, each node of the tree can be visited fewer times, increasing opportunities for optimization and decreasing cache pressure and other overheads. This fusion process is often done manually, requiring careful understanding of how each of the traversals of on tree interact.

This paper presents an automatic approach to traversal fusion: tree traversals can be written independently, and then our framework analyzes the dependences between the traversals to determine how they can be fused to reduce the number of visits to each node in the tree. A critical aspect of our framework is that it exploits two opportunities to increase the amount of fusion: i) it automatically integrates code motion, and ii) it supports partial fusion, where portions of one traversal can be fused with another, allowing for a reduction in node visits without requiring that two traversals be fully fused. We implement our framework as Clang tool, and show across several case studies that we can successfully fuse complex tree traversals, reducing the overall number of traversals and substantially improving locality and performance.

CCS Concepts: • Software and its engineering $\rightarrow$ Automated static analysis; Compilers;

Additional Key Words and Phrases: Locality Optimization, Fusion, Recursive Tree Traversals

ACM Reference Format:

Laith Sakka, Kirshanthan Sundararajah, and Milind Kulkarni. 2017. TreeFuser: A Framework for Analyzing and Fusing General Recursive Tree Traversals . Proc. ACM Program. Lang. 1, OOPSLA, Article 76 (October 2017), 30 pages. https://doi.org/10.1145/3133900

\section{INTRODUCTION}

Many key applications rely heavily on performing a series of tree traversals. For example, the DOM (Document Object Model) of a website is represented as a tree of elements, where each element contains a number of attributes specifying color, visibility, position, size, etc. The values of these attributes determine the appearance of a rendered web page. These attributes and elements are dependent on one another (for example, the width of an element is dependent on the widths and $\mathrm{x}$-positions of its children elements), so computing the appropriate values requires traversing the tree numerous times [Meyerovich et al. 2013]. Closer to home, many compilers use Abstract Syntax

Authors' Address: School of Electrical and Computer Engineering, Purdue University, 465 Northwestern Ave., West Lafayette, IN 47907, USA, \{lsakka,ksundar,milind\}@purdue.edu.

Permission to make digital or hard copies of all or part of this work for personal or classroom use is granted without fee provided that copies are not made or distributed for profit or commercial advantage and that copies bear this notice and the full citation on the first page. Copyrights for components of this work owned by others than the author(s) must be honored. Abstracting with credit is permitted. To copy otherwise, or republish, to post on servers or to redistribute to lists, requires prior specific permission and/or a fee. Request permissions from permissions@acm.org.

(C) 2017 Copyright held by the owner/author(s). Publication rights licensed to Association for Computing Machinery.

2475-1421/2017/10-ART76

https://doi.org/10.1145/3133900

Proc. ACM Program. Lang., Vol. 1, No. OOPSLA, Article 76. Publication date: October 2017. 
Trees (ASTs) as an intermediate representation, and compilation phases are expressed as traversals over these ASTs. Again, these traversals read and modify various attributes of the AST, and are dependent on one another (for example, a pass that performs constant folding may be dependent on a previous pass that performs type checking). Farther afield, the multiresolution simulation code MADNESS effectively performs multiple traversals over a kd-tree to perform various numerical simulations [Harrison et al. 2004].

An important consideration in these applications is to reduce the number of times the tree is traversed by fusing operations from separate traversals that operate on the same node. Fusion has numerous potential benefits: (i) if there is data reuse between operations from multiple traversals, fusion reduces reuse distance [Mattson et al. 1970] and can improve locality; (ii) increasing the amount of computation per node can expose opportunities for optimization; (iii) performing fewer traversals can lead to coarser-grain parallelism; (iv) if there are costs to accessing tree nodes-e.g., in distributed settings-reducing node accesses reduces overhead; (v) in functional settings, fusion is similar to deforestation [Wadler 1988], and avoids the materialization of temporary data structures. Hence, there has been substantial effort in applications that traverse trees to reduce the number of traversals.

In many cases, such as web browsers, what are conceptually multiple traversals of the tree are manually fused into fewer traversals. In other cases, traversals can be expressed using high level abstractions like attribute grammars (for compilers [Ekman and Hedin 2007] or DOM rendering [Meyerovich and Bodik 2010]) or numerical operators (in MADNESS [Harrison et al. 2004]) that can be fused using purpose-built frameworks that understand those abstractions (e.g., synthesisbased schedulers for attribute grammars [Meyerovich et al. 2013] or ad hoc high level compilers for MADNESS operators [Rajbhandari et al. 2016a,b]). These approaches suffer from various drawbacks: immense programmer effort and brittleness in the case of hand-written optimized traversals (adding a new DOM pass might require rewriting a large chunk of the renderer due to newly-introduced dependences), and the restrictions of high level abstractions in the latter (both in the inability of high level abstractions to capture more complicated traversal patterns and in the types of fusion decisions that can be made by the optimization framework).

Contributions. We want the best of both worlds: the ability to write simple, fine-grained traversals to express the computations required by an application, as in approaches using high level abstractions, while retaining the flexibility to perform more complicated traversal patterns as in hand-written code (e.g., performing computations between recursive calls). When these traversals are optimized and fused, we should retain the same capabilities as in hand-written optimizations. In particular, there are two optimization opportunities that are critical to exploit in order to maximize fusion that, to our knowledge, existing approaches do not explore:

(1) Code motion: By moving computations around within a traversal, fusion opportunities can be exposed that were previously disallowed. For example, moving a statement in a traversal from pre-order to post-order can allow it to be fused with other traversals. Code motion is especially important with fully-general traversals, where computations can be interleaved with recursive calls, as such in-order computations can introduce very complex dependence patterns.

(2) Partial fusion: Even if the computations performed by two traversals have complex dependences that preclude fusion, it may be possible to execute the traversals together on some parts of the tree but not others yielding some of the benefits of fusion without requiring complete fusion. As in the case of code motion, allowing general, complex traversals necessitates partial fusion, as the more-complicated dependence structure often precludes total fusion of multiple traversals. 
This paper presents a general method for traversal fusion of recursive tree traversal programs written in a general-purpose language. This method provides several novelties: (a) a dependence graph representation of traversals that captures interactions between statements and calls; (b) a dependence test that uses the dependence graph to determine which fusion opportunities are legal and supports partial fusion and code motion; and (c) a synthesis procedure for fused traversals that naturally implements code motion.

We implement our method in a framework called TreeFuser, a Clang source-to-source compiler pass, allowing programmers to express their set of tree traversals using (a subset of) $\mathrm{C}++$. The pass automatically lifts the tree traversals to TreeFuser's dependence representation, and then uses a simple greedy heuristic to synthesize a new, fused implementation of the set of traversals, including moving computations to remove problematic dependences and performing partial fusion when necessary.

To evaluate the suitability of TreeFuser for enabling fine-grained expression of general tree traversals while supporting fusion, we evaluate several case studies: fusing together passes in Fast Multipole Method, fusing passes over a DOM rendering tree, and fusing complex AST passes in a compiler. In all three cases, we demonstrate that TreeFuser is able to successfully fuse a substantial portion of the computation, requiring both code motion and partial fusion to do so. The fused traversals substantially improve locality and overall performance.

\section{RELATED WORK}

Fusion in various settings. Fusion and similar transformations form the basis for many crucial compiler optimizations. In the world of regular programs (programs that operate over dense matrices and arrays), loop fusion is a common optimization that improves locality (by reducing the number of times that an array or matrix is accessed) by merging the bodies of two (or more) loops together [Darte 1999; Kennedy and McKinley 1994; Qasem and Kennedy 2006]. Perhaps the most popular framework for performing fusion is the polyhedral framework [Bondhugula et al 2008; Feautrier 1992a,b], which uses a high level dependence representation that allows compilers to reason about the dependences between loops and explore different fusion possibilities prior to synthesizing code to implement a fused schedule. These approaches are not suitable for traversal fusion, as the dependence representation applies to loops over arrays with affine access functions.

There is a close connection between traversal fusion and deforestation in functional programs [Chin 1992; Gill et al. 1993; MartÃČÂnnez and Pardo 2013; Wadler 1988], which combines a series of operations on a data structure into a single composed operation, eliminating intermediate structures. D'Antoni et al. [2014] specifically look at deforestation in programs that manipulate trees. While deforestation originally adopted a syntactic, rewrite-based approach, later work focused on providing deforestation benefits by translating programs that traverse data structures to use combinators with known fusion rules (Hinze et al. [2011] provide a survey). For example, fusion rules for monadic folds [Meijer and Jeuring 1995] handle fusing operations that maintain state.

While deforestation techniques are quite powerful, they are insufficient in various ways in our setting of fusing more general imperative traversals. Syntactic approaches to fusion cannot handle the complex types of dependences that can arise in our setting (as reasoning about them would require some form of dependence analysis), while combinator-based approaches make it hard to express arbitrary traversal orders and arbitrary manipulations of fields during that traversal. Moreover, to our knowledge, TreeFuser's partial fusion and code motion optimizations do not have analogs among most deforestation techniques. Rompf et al. [2013] implemented a variant of deforestation in a compiler that also integrates code motion, but their fusion rules cannot capture the complexities of fusion over tree traversals. 
An alternative to performing fusion over general code is to express traversals using a high-level abstraction and then design optimization frameworks that work on that high-level abstraction to optimize traversals. One popular abstraction for capturing traversals of trees is attribute grammars [Knuth 1968]. In recent work, Meyerovich and Bodik [2010] showed how complex web page rendering passes can be expressed using attribute grammars and then scheduled using a combination of fusion and parallelization [Meyerovich et al. 2013]. While their approach is highly effective, attribute grammars are restrictive in the kinds of traversals they can express: a single complex traversal might require a careful decomposition into several interlocking attributes. Moreover, existing attribute grammar scheduling frameworks do not support partial fusion.

Attribute grammars are a special case of tree transducers, which are automata that recognize tree languages (equivalently, traverse trees) and produce an output as they go [Comon et al. 2007; Doner 1970]. Macro tree transducers allow for more arbitrary computations by allowing the transducer to accumulate context as it traverses the tree [Engelfriet and Vogler 1985]. There has been significant investigation into the composition of various types of tree transducers [Engelfriet 1975; Engelfriet and Maneth 2002; Maletti 2008]. Tree transducers, like attribute grammars, require expressing traversals in a particular formalism, in contrast to our more natural, code-like expression. Moreover, because our language for fusion admits fairly general computations and interleaving of computation and traversal, we believe that our approach covers a more general class of problems than tree transducers, though this requires further study. Finally, to our knowledge, there is no analog in transducer composition to our notion of partial fusion.

Rajbhandari et al. [2016a,b] looked at fusing kd-tree traversals in the MADNESS simulation package. They capture the various kd-tree passes in MADNESS using a high level representation and then reason about when fusion is legal using a dependence test similar to that of Weijiang et al. [2015]. Rajbhandari et al.'s abstraction only supports pre- and post-order traversal, requires that every node in the traversed tree have the same structure, and requires that each traversal be compatible (i.e., that each traversal visit all the children of a node), and hence is not expressive enough to handle, e.g., arbitrary AST traversals. As a result of these limitations, their optimization framework also does not handle general code motion, and does not support partial fusion.

Petrashko et al. [2017] described miniphases, an approach to architecting a compiler built around fusing small passes over abstract syntax trees. Their approach is similar in spirit to deforestation using combinators: by requiring that AST passes be structured in a certain way, they can implement a pre-determined fusion strategy. Crucially, however, that their approach does not provide any soundness guarantees: it is up to the programmer to affirm that a particular sequence of fusion passes will not violate any dependences.

Analysis and transformation of irregular programs. A line of more generally related work is in the analysis and optimization of irregular programs. Madhusudan et al. [2012] investigated verifying properties of recursive programs (including tree programs), but did not consider transformations. Ghiya et al. [1998] analyzed accesses to tree data structures to determine whether calls to different children were independent. Larus and Hilfinger [1988] used access paths to determine whether two accesses to a pointer-based data structure might interfere. Rugina and Rinard [2005] use symbolic expressions to determine whether two pointer dereferences might interfere with one another. Most analyses for irregular programs concern themselves primarily with independence and parallelization, and hence are not suitable for traversal fusion, which must take into account the structure of dependences. In other words, we must perform dependence analysis, not dependence detection.

One way to perform dependence analysis of recursive programs (such as tree traversals) is to perform an instance-wise analysis, that determines dependences between specific dynamic instances 
of statements, rather than between static statements [Amiranoff et al. 2006; Cohen and Collard 1998; Feautrier 1998].

Weijiang et al. [2015] used an access-path-based representation of these instance-wise dependences to prove the correctness of tiling transformations on trees. Weijiang et al.'s analysis and transformation assumed that all traversals were identical, and only handled preorder recursive traversals. Moreover, their dependence analysis considered the relation between dynamic instances of entire function bodies, because they treated each function invocation as a single unit of computation. Hence, their analysis did not build a dependence graph and could not capture the fine-grained, statement-to-statement dependences required to perform code motion or partial fusion.

\section{OVERVIEW}

This section provides an overview of the TreeFuser framework. We first discuss the opportunities for fusion in a running example (Section 3.1). We then describe how TreeFuser constructs a dependence representation for the example (Section 3.2). We then explain how TreeFuser uses the dependence representation to construct a fused traversal, including partial fusion, and show the final synthesized result (Section 3.3). Each of these steps are described in more detail in the remainder of the paper.

\subsection{Motivating Example}

Figure 1(a) shows two traversals, $\mathrm{f} 1$ and $\mathrm{f} 2$, over a binary tree (the argument $\mathrm{n}$ to each traversal represents the node the traversal is currently visiting). While the traversals perform somewhat complex computations, we can consider each as a sequence of statements, where compound statements such as if statements are represented as single statements, interspersed with recursive invocations of the traversal function that traverse the tree further. Hence, we can consider $\mathrm{f} 1$ to perform one statement per node during its traversal, and $\mathrm{f} 2$ to perform two, as shown by the gray outlined boxes. (For now, we ignore the $\mathrm{n}=$ null line of each traversal as that serves simply to bound the traversals; later we will explain how to soundly handle such conditions.)

Suppose the two traversals visit the tree shown in Figure 1(b), with $\mathrm{f} 1$ executing before $\mathrm{f} 2$ leading to the sequence of statements shown in Figure 1(c). Each statement is parameterized by which node of the tree it operates on, and arrows between statements indicate dependences. For example, $s_{1}(b)$ writes to b.v, which is read by $s_{1}(a), s_{2}(b)$ and $s_{3}(b)$. The vertical line separates the operations performed by the two traversals. As we see, the tree is traversed twice, and, in particular, each leaf node is visited twice.

Opportunity for fusion. Careful examination of the dependence structure shows that it may be possible to perform operations from both traversals while visiting some of the nodes in the tree. In particular, all of the operations on node c can be performed when the first traversal visits node c (in other words, $s_{2}(c)$ and $s_{3}(c)$ can be hoisted up to execute immediately after $s_{1}(c)$ ). As a result, the second traversal need not visit the right child of the tree at all, saving one node visit. Note, however, that the two visits to node $b$ must happen separately: $s_{1}$ must execute on $b$ before executing on $a$, but $s_{2}$ must execute on a before executing on $b$. The only way to achieve this is by visiting $b$ twice. We will now see how TreeFuser identifies this opportunity for fusion.

\subsection{Dependence Representation}

The dependences between the various operations of two traversals is a dynamic property: two statements depend on each other depending on where, in a particular tree, the two statements are being executed. To avoid reasoning about (unknowable) dynamic iterations, TreeFuser uses a dependence graph representation that captures dependences between static instances of statements based on a dependence analysis. Section 4.3 details how this dependence graph is constructed. In 


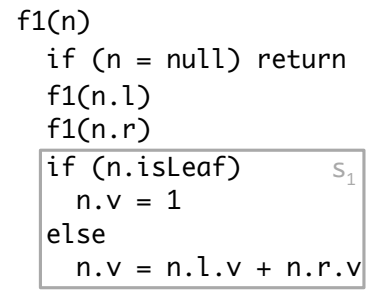

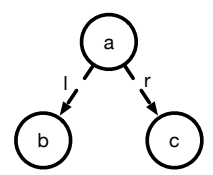

(b) Example binary tree with three nodes

(a) Two traversals of a binary tree

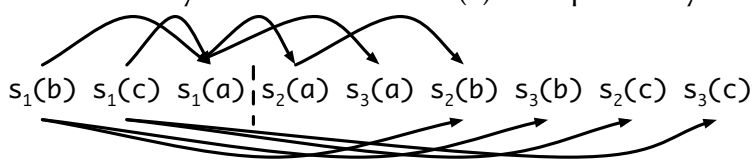

(c) Original schedule of execution, with dependences

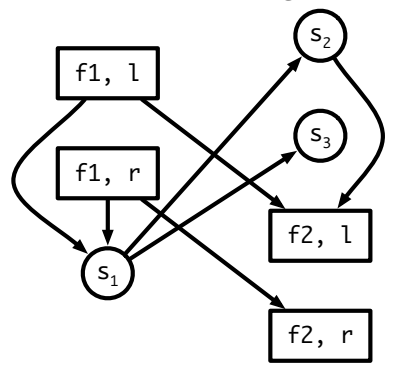

(d) Dependence graph, pre-fusion

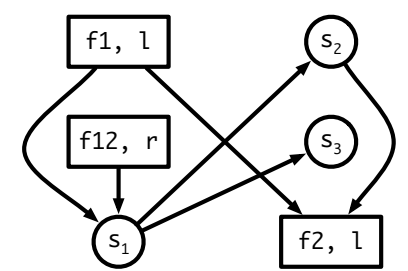

(e) Dependence graph, post-fusion

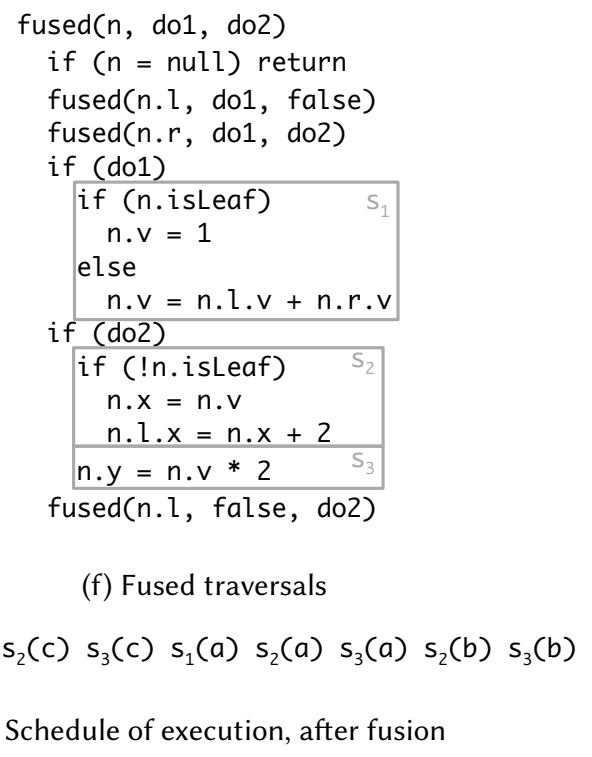

Fig. 1. Running example: two traversals over a binary tree 
short, each (compound) statement and recursive call in the original traversals is represented by a node in the dependence graph. Statement nodes are labeled by which traversal they are from and which (static) statement in that traversal they represent, while call nodes are labeled by which traversal they are from and by which child that call descends to.

Figure 1(d) shows the dependence graph for the statements of the traversals in Figure 1(a). The graph considers the execution of each traversal rooted at the same (arbitrary) node in the tree. Directed edges exist between statements if there may be dependences between those statements when executed at that node. Hence, the dependence between $\mathrm{s}_{1}(\mathrm{a})$ and $\mathrm{s}_{2}(\mathrm{a})$ leads to an edge in the dependence graph between the node $s_{1}$ and the node $s_{2}$. Edges exist between statements and calls if there may be a dependence between the statement and statements that may execute during that call. Hence, the dependence between $s_{1}(b)$ and $s_{1}(a)$ leads to an edge between the call node $f 1, I$ and the statement node $s_{1}$. Similarly, edges exist between calls if there may be a dependence between the statements that may execute during those calls. The direction of the edges corresponds to the execution of the original, unfused program: between two nodes from the same traversal, the edge is directed in program order, and between two nodes from different traversal, the edge is directed according to the order of the traversals. Essentially, the dependence graph in Figure 1(d) represents taking the execution graph of Figure 1(c) and collapsing the various vertices for nodes $\mathrm{b}$ and $\mathrm{c}$ into call nodes representing the corresponding recursive call.

\subsection{Fusion and Code Generation}

Two calls to the same child of a tree node are fusable if it is safe for two traversals to visit that child simultaneously (rather than one traversal completely processing that child subtree before the second traversal visits it). Two traversals are fully fusable if all of their child calls are fusable. If only some child calls are fusable, the two traversals are partially fusable. The dependence graph makes it easy to tell if two calls are fusable: if the two call nodes in the graph can be merged without creating a cycle, then the nodes are fusable. Section 7 argues that this validity test is sound.

In our running example, we can see that fusing the two calls to the left child results in a cycle involving $s_{1}$ and $s_{2}$. However, fusing the two calls to the right child does not create a cycle, yielding the dependence graph in Figure 1(e).

Given a fused dependence graph, TreeFuser synthesizes the fused traversal by topologically sorting the dependence graph and then generating code as if the resulting graph were for a single traversal function (including guards to make sure that a statement only executes if its original traversal would have executed). Figure 1 (f) shows the resulting code. Note that this synthesis procedure automatically incorporates code motion: in the fused, sorted graph, $s_{3}$ appears after the traversal visits the right child, whereas in the original traversal, $s_{3}$ executed prior to its traversal visiting the right child. Section 5 explains this process in more detail.

The final traversal produces the schedule of execution shown in Figure $1(\mathrm{~g})$. This new schedule preserves all dependences-dependent operations appear in the same relative order as they did in the schedule of Figure 1(c)-but now node c is only visited once, providing the benefits of fusion (this fused traversal is valid for any tree, and this reduction in visits grows as the tree grows). The remainder of this paper explains how TreeFuser achieves this result.

\section{A DEPENDENCE ANALYSIS FOR FUSION}

This section presents a dependence analysis for fusion. It is a variant of access-path-based dependence analyses, such as those used by Larus and Hilfinger [1988] and Hummel et al. [1994]. Because we adopt an instance-wise notion of accesses [Cohen and Collard 1998] (that is, we consider accesses relative to a particular node that a traversal is visiting), our dependence test is informed 


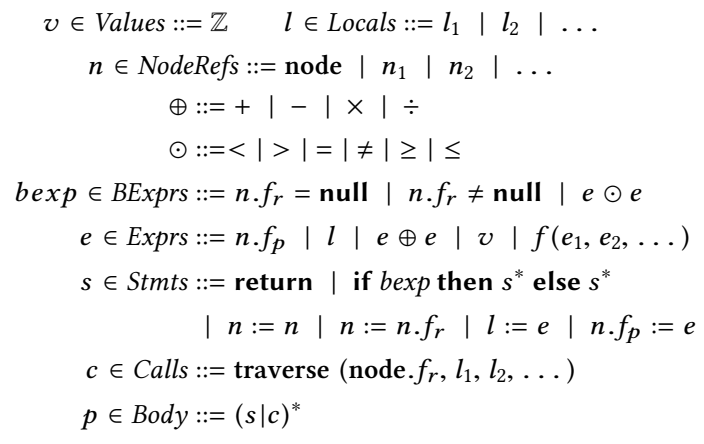

(a) Language for defining tree traversal

$$
\begin{aligned}
& \mathbf{f}_{1}\left(\operatorname{root}, l_{1}, l_{2}\right) \\
& \mathbf{f}_{2}\left(\operatorname{root}, l_{3}, l_{1}\right) \\
& \mathbf{f}_{3}\left(\operatorname{root}, l_{4}, l_{5}\right)
\end{aligned}
$$

(b) Example series of initial traversal calls

Fig. 2. Defining tree traversal programs

by that of Weijiang et al. [2015]. First, we present a simple (but general) language for expressing tree traversals that we will use to build our dependence analysis.

\subsection{A Language for Tree Traversals}

A tree that is being traversed consists of several tree nodes. Each tree node contains one or more recursive fields $\left(f_{r} \in \mathcal{F}_{r}\right)$ that point to other tree nodes (we will often refer to these recursive fields as the "children" of the tree node), and zero or more local fields $\left(f_{p} \in \mathcal{F}_{p}\right)$. These local fields can be simple primitive values or can be pointers to node local objects (objects that can only be reached through that local field). The nodes are arranged in a tree, connected through the recursive fields, and rooted at a single root node ${ }^{1}$.

A tree is traversed by recursive traversal functions whose bodies can be expressed in the language given in Figure $2(a)^{2}$. Each function is $k$-ary: the first parameter of the function is a reference to the node the traversal operates on; the rest of the parameters are local variables.

The body of the traversal function is a series of (compound) statements and recursive calls. Each statement can be a single primitive statement (an assignment or a return) or an if statement that can then be arbitrarily nested. Expressions can dereference fields of node and read local variables, and can invoke pure functions. Assignments can update local variables and primitive fields of node (note that the recursive fields of node cannot be modified). A recursive call takes one of the recursive fields of node as its first argument and local variables as its remaining arguments (we will often refer to these recursive calls as "child calls," as they are invoked on specific children of the current node).

A few things to note about this language:

(1) We do not allow loops in the body of the recursive function (unless those loops can be statically unrolled).

(2) Recursive calls do not return values; return values can be de-sugared as writing to a primitive field of node in the callee, then reading from that primitive field (via the appropriate recursive field) in the caller.

(3) Recursive calls cannot be made conditionally. This can be worked around by conditionally setting a local variable that is passed into the recursive call that causes the call to exit immediately.

\footnotetext{
${ }^{1}$ Note that our analysis does not concern itself with proving these properties of the tree; we leave this to shape analyses [Ghiya and Hendren 1996; Sagiv et al. 1999]. The implementation in Section 8 relies on programmer- or shape analysis-provided annotations to convey these properties.

${ }^{2}$ TreeFuser analyzes programs written in $\mathrm{C}++$, but that respect the constraints of this language
} 
(4) Interestingly, the recursive invocations of the traversal function a) do not have to be invoked on every child; and b) can be invoked on a child more than once. In other words, a recursive traversal function may not visit all children of a node, and may visit the same subtree multiple times.

A program analyzed by TreeFuser is a sequence of calls to traversal functions defined using the language of Figure 2(a), all starting at root (Figure 2(b)). We do not consider the existence of intervening code between successive calls to traversal functions; handling any dependences that arise from such code is a straightforward extension of the analyses in this paper.

Handling different child types. The language of Figure 2(a) assumes that all tree nodes are the same type. This assumption is violated by many tree traversal codes (e.g., the multitude of node types in a typical AST). We currently handle this case by merging all the node types into a single node type (a la a tagged union), and using conditionals to distinguish between the code that should run in each node type. If a recursive call is made to a child that does not exist for the current node type, that call sees that the child node is null and returns immediately. This transformation can be done automatically, though it reduces the precision of our analysis (since we cannot account for which node types perform which computations when determining dependences).

\subsection{Access-path Analysis}

The first step in TreeFuser's dependence analysis is to perform an analysis to construct access sets for each compound statement and call in each recursive traversal function.

We abstract the "off-tree" locations that can be accessed by functions as a set of heap locations $h \in \mathcal{H}$-these correspond to whatever representation a (separately run) alias analysis uses to represent heap locations. We represent accesses to the nodes and fields of the tree using access paths. A recursive access path is a path to a node rooted at node. These access paths can be represented by elements of the regular set $\mathcal{A}_{r}=\operatorname{node}\left(. \mathcal{F}_{r}\right)^{*}$. A primitive access path is a path rooted at node terminating in a primitive field, and can be represented by elements of the regular set $\mathcal{A}_{p}=\operatorname{node}\left(. \mathcal{F}_{r}\right)^{*} \cdot \mathcal{F}_{p}$.

Statements. Each compound statement $s$ is associated with four sets: a set of heap locations read and written $\left(H_{r}(s)\right.$ and $H_{w}(s)$ respectively) and a set of primitive access paths read and written $\left(\pi_{r}(s)\right.$ and $\pi_{w}(s)$ respectively). Heap locations read and written by a statement can be computed via a straightforward dataflow analysis on the body of the function, treating function calls as no-ops. Unioning together all of the reads and writes of the component statements of a compound statement yields $H_{r}(s)$ and $H_{w}(s)$.

Generating $\pi_{r}(s)$ and $\pi_{w}(s)$ is similar to other analyses [Larus and Hilfinger 1988; Weijiang et al. 2015; Wiedermann and Cook 2007], so we treat it briefly here (essentially, we perform an alias analysis on node references, using an access-path-based representation). Access paths read and written are computed by an alias analysis that tracks the (set of) recursive access paths referred to by node references. Assignments to node references generate new recursive access paths for that node reference in the obvious way (maintaining multiple possible access paths for the left hand side of the assignment if the right hand side maps to multiple paths). Assignments to primitive fields of a node reference create write access paths by appending the primitive field to the access path(s) of the node reference, and uses of primitive fields in expressions create read access paths by appending the primitive field to the access path of the node reference. Conditionals in if statements can generate read accesses, but are otherwise not evaluated; the analysis processes both branches of the conditional. Access paths associated with node references are joined in if statements using set 
union. For a compound statement $s, \pi_{r}(s)$ is the union of all the read access paths in the components of the compound statement, and $\pi_{w}(s)$ is the union of all the write access paths.

Calls. Each call $c$ contains summaries of all the reads and writes that could be performed during the invocation of the call (including further recursive invocations). Summarizing the heap accesses is straightforward. A call's heap read and write sets $\left(H_{r}(c)\right.$ and $\left.H_{w}(c)\right)$ are the union of the method body's statement's heap read and write sets. Note that because heap locations cannot be accessed via fields of the tree, the set of possible heap locations accessed by the recursive invocation is exactly the same as the caller's.

Summarizing a call's accesses to the tree is subtle. Because a recursive call can make additional recursive calls, each access to the tree can repeat, each time being rooted at a different node. To capture this information, we define an extended access path (this follows the approach of Larus and Hilfinger [1988]). Rather than being a member of the regular set $\mathcal{A}_{p}$, an extended access path is, itself, a regular set.

To construct the extended access paths for a particular call, traverse (node. $r_{1}, \ldots$ ), we first construct the base read (respectively, write) set of access paths by unioning all the read (respectively, write) access path sets of the statements in the method body. Consider a specific primitive access path in the read set: node. $r_{a} . r_{b} . p_{a}$. The extended access path for this path for this call is: node. $r_{1}\left(. \mathcal{F}_{r}\right)^{*} \cdot r_{a} \cdot r_{b} \cdot p_{a}$. In other words, we take the suffix of the access path and insert a regular set consisting of the child the recursive call descends to (which represents the root of the access paths in the immediate callee) followed by zero or more additional children (which represents any subsequent recursive calls made by the method) $)^{3}$. By constructing extended access paths for each base access path for a call, we can obtain a set of regular sets whose union (over) approximates all of the fields of the tree that recursive call might access.

Running example. In our running example (Figure 1(a)), there are no heap accesses, so we need only consider access paths. We can obtain the following access paths for the statements (note that in our example, $n$ represents node):

$$
\begin{aligned}
& \pi_{r}\left(s_{1}\right)=\{n . l . v, n . r . v\} \\
& \pi_{r}\left(s_{2}\right)=\{n . v, n . x\} \\
& \pi_{r}\left(s_{3}\right)=\{n . v\}
\end{aligned}
$$

$$
\begin{aligned}
& \pi_{w}\left(s_{1}\right)=\{n . v\} \\
& \pi_{w}\left(s_{2}\right)=\{n . x, n . l . x\} \\
& \pi_{w}\left(s_{3}\right)=\{n . y\}
\end{aligned}
$$

The extended access paths for the calls are:

$$
\begin{aligned}
& \pi_{r}\left(f_{1}(n . l)\right)=\left\{n . l(.(l \mid r))^{*} . l . v, n . l(.(l \mid r))^{*} . r . v\right\} \\
& \pi_{w}\left(f_{1}(n . l)\right)=\left\{n . l(.(l \mid r))^{*} . v\right\} \\
& \pi_{r}\left(f_{1}(n . r)\right)=\left\{n . r(.(l \mid r))^{*} . l . v, n . r(.(l \mid r))^{*} . r . v\right\} \\
& \pi_{w}\left(f_{1}(n . r)\right)=\left\{n . r(.(l \mid r))^{*} . v\right\} \\
& \pi_{r}\left(f_{2}(n . l)\right)=\left\{n . l(.(l \mid r))^{*} . v, n . l(.(l \mid r))^{*} . x\right\} \\
& \pi_{w}\left(f_{2}(n . l)\right)=\left\{n . l(.(l \mid r))^{*} . x, n . l(.(l \mid r))^{*} . l . x, n . l(.(l \mid r))^{*} \cdot y\right\} \\
& \pi_{r}\left(f_{2}(n . r)\right)=\left\{n . r(.(l \mid r))^{*} . v, n . r(.(l \mid r))^{*} . x\right\} \\
& \pi_{w}\left(f_{2}(n . r)\right)=\left\{n . r(.(l \mid r))^{*} \cdot x, n . r(.(l \mid r))^{*} \cdot l \cdot x, n . r(.(l \mid r))^{*} \cdot y\right\}
\end{aligned}
$$

\footnotetext{
${ }^{3}$ This is made more precise by using the subset of recursive fields that are actually visited by the traversal function in question, rather than $\mathcal{F}_{r}$
} 


\subsection{Building a Dependence Graph}

We are now prepared to build the dependence graph to capture dependences between statements and calls, which can help determine when fusion is legal. The key to the dependence graph is that it captures the execution of every statement and call across the set of traversals, $T$, considered for fusion assuming they are executing at the same node in the tree. This is because the goal of fusion is to merge computations at the same node of the tree together so that the two traversals can occur simultaneously.

The dependence graph is a directed graph $G=(V, E)$. The graph contains a vertex for each (compound) statement and call in the set of traversal functions $T$. Every vertex is labeled using a labeling function $l: V \rightarrow T \times\left(\{s\} \cup \mathcal{F}_{r}\right)$. In other words, a vertex is labeled with a tuple whose first element is the traversal function the vertex is from, and whose second element denotes whether the vertex is a statement (labeled $s$ ) or a call (labeled with the recursive field of node that the call descends to).

The edges in the graph are directed, and capture data and control dependences between statements and calls, both within traversals and across traversals.

For every pair of vertices $v_{1}$ and $v_{2}$, where $v_{1}$ appears before $v_{2}$ in program order (either they are in the same traversal and $v_{1}$ is earlier in the traversal, or $v_{1}$ appears in an earlier traversal than $\left.v_{2}\right),\left(v_{1}, v_{2}\right) \in E$ iff one or more of the following is true:

(1) $v_{1}$ and $v_{2}$ are part of the same traversal and there is a dependence through local variables between $v_{1}$ and $v_{2}$. This can be established through a straightforward intra-procedural reaching-definitions analysis.

(2) $v_{1}$ and $v_{2}$ are part of the same traversal and $v_{1}$ is a compound statement that contains a return statement. In this case, $v_{2}$ is control dependent on $v_{1}$, as if $v_{1}$ returns, $v_{2}$ will not execute. On the other hand, if $v_{2}$ contains a return statement, there will be a control dependence from $v_{1}$ to $v_{2}$ to ensure that $v_{1}$ does execute.

(3) $H_{w}\left(v_{1}\right) \cap\left(H_{r}\left(v_{2}\right) \cup H_{w}\left(v_{2}\right)\right) \neq \emptyset$ or $H_{w}\left(v_{2}\right) \cap\left(H_{r}\left(v_{1}\right) \cup H_{w}\left(v_{1}\right)\right) \neq \emptyset$. In other words, the two statements/calls access the same heap location, and at least one of them is a write.

(4) $v_{1}$ and $v_{2}$ are both statements, and $\pi_{w}\left(v_{1}\right) \cap\left(\pi_{r}\left(v_{2}\right) \cup \pi_{w}\left(v_{2}\right)\right) \neq \emptyset$ or $\pi_{w}\left(v_{2}\right) \cap\left(\pi_{r}\left(v_{1}\right) \cup\right.$ $\left.\pi_{w}\left(v_{1}\right)\right) \neq \emptyset$. That is, if $v_{1}$ and $v_{2}$ are both statements, there is an edge between them if they share an access path, with at least one of those accesses being a write.

(5) $v_{1}$ (resp. $v_{2}$ ) is a call and its extended access path(s) collide with the (extended) access paths of $v_{2}$ (resp. $v_{1}$ ). The notion of collision is subtle. Assume, without loss of generality, that $v_{1}$ is a call. For each extended access path $a_{1} \in \pi_{w}\left(v_{1}\right)$, we intersect $a_{1}$ with each (extended) access path in $\pi_{r}\left(v_{2}\right) \cup \pi_{w}\left(v_{2}\right)^{4}$, and test for the emptiness of the resulting regular set. If any such set is non-empty, then $v_{1}$ collides with $v_{2}$. We can perform a similar procedure to check for the scenario where $v_{2}$ performs the write and $v_{1}$ performs any colliding access.

The resulting dependence graph captures the ordering between statements and calls that must be respected by any attempted traversal fusion: if two statements are to execute at the same node in the tree at the same time, and there is a dependence edge between them, they must execute in an order that respects that dependence. Note that the dependence graph is guaranteed to be acyclic (since the edges are directed in program order).

Running example. Figure 1(d) shows the dependence graph for our running example, leaving out the null test at the beginning of each traversal. Each null test results in control dependences between that test and all of the other statements/calls in its traversal.

${ }^{4}$ If $v_{1}$ is a statement, we can treat its access paths as singleton regular sets. 


\section{TRAVERSAL FUSION}

This section explains how TreeFuser uses the dependence graph to determine whether fusion is legal.

\subsection{Traversal Fusion as Child-call Fusion}

Fusing together two traversals means executing operations from multiple traversals while visiting a node of a tree, rather than visiting the node separately for each traversal. We can phrase the problem of fusing traversals as a problem of fusing child calls. Suppose two traversals start at the same node (e.g., the root node). If we can combine recursive calls to the same child from the two traversals, then a single visit to that child will accomplish the work of both traversals (note that this does not mean the total number of visits is cut in half; after descending to the child, the traversals may diverge upon further recursive calls).

Viewing fusion as fusing child calls is a generalization of "total" fusion. Suppose we have two traversals, $f_{1}$ and $f_{2}$ that operate over a binary tree. Hence, each traversal has two recursive calls, one visiting node. $l$ and another visiting node. $r$. If the two traversals' calls to node. $l$ are fused and their calls to node. $r$ are fused, then we have achieved total fusion: the fused function will traverse the tree just once, and at each child call will do the work of both $f_{1}$ and $f_{2}$. If only some of the children are fused, then we have achieved "partial" fusion. In Figure 1(f), the calls to the right child are fused together, but the calls to the left child are not. If both traversals are at the same node, they will both descend to the right child together, but will process the left child separately. In general, then, the more recursive calls we can fuse together, the fewer times we will visit nodes in the tree. Indeed, TreeFuser's goal is not to "fuse traversals." Instead, its goal is to fuse together multiple recursive calls.

\subsection{Dependence Test and Fusion}

TreeFuser constructs a dependence graph as outlined in Section 4.3. This dependence graph can then be used to test whether two calls can be fused together.

First, we slightly modify the labeling function for the graph. Rather than mapping vertices to traversals and types $\left(l: V \rightarrow T \times\left(\{s\} \cup \mathcal{F}_{r}\right)\right)$, the labeling function maps vertices to sets of traversals and types $\left(l: V \rightarrow \mathcal{P}(T) \times\left(\{s\} \cup \mathcal{F}_{r}\right)\right)$. The initial dependence graph's vertices are all labeled with single-element traversal sets.

Then, we define an operation called fuse, which takes an acyclic graph, $G=(V, E)$, and two call vertices, $v_{1}, v_{2} \in V$, where $l\left(v_{1}\right)=\left(T_{1}, r_{1}\right)$ and $l\left(v_{2}\right)=\left(T_{2}, r_{1}\right)$ (i.e., both vertices are calls that descend to the same child). fuse $\left(G, v_{1}, v_{2}\right)$ produces a new graph $G^{\prime}=\left(V^{\prime}, E^{\prime}\right)$ with:

$V^{\prime}$ is $V$ with $v_{1}$ and $v_{2}$ removed, and a new vertex $v_{12}$ added, where $l\left(v_{12}\right)=\left(T_{1} \cup T_{2}, r_{1}\right)$

$E^{\prime}$ retains all edges in $E$ that are not incident on either $v_{1}$ or $v_{2}$, removes any edge between $v_{1}$ and $v_{2}$, and for edges incident on $v_{1}$ (or $v_{2}$ ), replaces $v_{1}$ (resp. $v_{2}$ ) with $v_{12}$.

$G^{\prime}$ represents fusing the calls $v_{1}$ and $v_{2}$. We can define fusability as follows:

Definition 5.1. Two calls, $v_{1}$, where $l\left(v_{1}\right)=\left(T_{1}, r_{1}\right)$, and $v_{2}$, where $l\left(v_{2}\right)=\left(T_{2}, r_{1}\right)$, are fusable iff $\operatorname{fuse}\left(G, v_{1}, v_{2}\right)$ is acyclic.

The soundness proof for this definition is in Section 7, but the intuition is as follows. Dependence edges between vertices in $G$ require that the vertices execute in that order to preserve either data or control dependence. Merging together two call vertices (i.e., merging together two calls) ensures that those calls will happen simultaneously, so the two visits to the child will occur at the same time. Any other vertices that are dependent on either of those two calls will still need to respect 


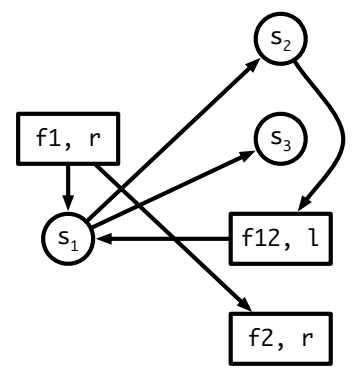

Fig. 3. Invalid dependence graph, resulting from attempted fusion of left children in Figure 1(d).

their original dependences, and if there is a cycle in the merged graph, there is no program order that can respect all dependences.

Note that fusion does not require that the two child calls fused together come from different traversals. In some settings, it may be more natural to conceive of a traversal operation as visiting the same subtree multiple times, even though it is possible to implement the operation with a single visit to a subtree. In such cases, a programmer can write the "multiple subtree" version of the traversal and rely on fusion to merge the subtree visits together.

Fusing together two child calls produces $G^{\prime}$, and the process of fusing together further child calls can be repeated recursively. The operation fuse is the fusion mechanism; choosing which child calls to apply it to is a policy decision (see Section 8) and different fusion policies might result in different fused graphs.

Running example. Figure $1(\mathrm{~d})$ is the dependence graph for our running example. There are two possible options for child fusion here: the two calls to the left child (labeled $\left(f_{1}, l\right)$ and $\left(f_{2}, l\right)$ ), and the two calls to the right child (labeled $\left(f_{1}, r\right)$ and $\left(f_{2}, r\right)$ ). Figure $1(\mathrm{e})$ shows the result of fusing the two right children, producing a new merged node $\left(f_{12}, r\right)$. Here we see that there are no cycles, so this is a valid fusion option. Had we tried to fuse the two left children, we would have obtained the dependence graph in Figure 3, producing a cycle, which is an invalid fusion. Intuitively, this is invalid because the first traversal needs to visit its left child before computing n.v $=$ n.l.v + n.r.v, while the second traversal needs to compute n.l.x $=n . x+2$ before visiting its left child. If both traversals descend to the left child at the same time, one traversal will be unable to perform its computations at the correct time.

\section{SYNTHESIZING A FUSED TRAVERSAL}

This section describes how TreeFuser synthesizes a tree traversal from a dependence graph, taking into account any fused child calls. The key is generating a single tree traversal function, regardless of how many original traversals are represented in the dependence graph, and regardless of how many child calls are fused. We begin by presenting a basic traversal template that generates a valid single traversal for the unfused dependence graph (Section 6.1). We then show how this same template can be modified to generate traversals for merged children (Section 6.2). Finally, we explain how this synthesis procedure naturally and implicitly incorporates any required code motion (Section 6.3).

Note that while fusion is a valid operation on any recursive calls to the same child, whether from different traversals or from the same traversal, the synthesis procedure presented here only applies to fusion of calls from different traversals. 


\subsection{Traversal Template}

TreeFuser generates a single traversal function from an (unfused) dependence graph through rewrite rules.

Consider a dependence graph generated from a set of traversals $T=\left\{t_{1}, t_{2}, \ldots\right\}$. The signature of the new traversal function is:

$$
\text { traverse }_{f}\left(\text { node }, o_{1}, o_{2}, \ldots, b_{1}, b_{2}, \ldots\right)
$$

Where $o_{1}, o_{2}$, etc., are pointers to (local) structures that contain fields corresponding to the local variable parameters of traversals $t_{1}, t_{2}$, etc. (assume, without loss of generality, that the names of the local variables in all of the traversals are unique). ${ }^{5}$ The variables $b_{1}, b_{2}$, etc., are boolean variables corresponding to each traversal. Intuitively, $b_{1}, b_{2}$, etc., determine whether a particular traversal is "active" during a given visit to a node (i.e., during a given execution of the traversal function). The overall traversal is called by initializing the $o$ objects to the appropriate local variables, calling traverse on the root node, and passing in true for all of the boolean variables (i.e., at the root, all traversals are active).

The next step is filling in the body of the traversal function. The first part of the traversal function extracts all the local variables from (non-nulI) objects passed into traverse $f_{f}$ and stores them in local variables with the same names as in the original traversals.

TreeFuser then generates a topological sort of the dependence graph to determine the order statements/calls should appear in the body. It then inserts the statements and calls into the body one by one.

Statements. For each statement $s_{i}$ with vertex label $\left(\left\{t_{j}\right\}, s\right)$, TreeFuser generates the following code:

$$
\begin{aligned}
& \text { if }\left(b_{j}=\text { true }\right) \text { then } s_{i}^{\prime} \\
& \text { label } i:
\end{aligned}
$$

where $s_{i}^{\prime}$ is $s_{i}$ with all return statements replaced with

$$
b_{j}=\text { false; } \text { goto } i
$$

Recall that $b_{j}$ is the variable that determines whether traversal $t_{j}$ is meant to execute during this visit to a node. Hence, $s_{i}$, which comes from traversal $t_{j}$ should only execute if $b_{j}$ is true. Moreover, if $s_{i}$ calls return, the traversal should truncate at this tree node and not descend to any further children. This is achieved by having the traversal turn itself off by setting $b_{j}$ to false, and then skip over the remainder of $s_{i}$ (with the goto statement).

Calls. For each recursive call vertex label $\left(\left\{t_{j}\right\}, f_{r}\right)$, (i.e., a recursive call that looks like: traverse (node. $\left.f_{r}, l_{j_{1}}, l_{j_{2}}, \ldots\right)$ ), TreeFuser generates the following code:

$$
\begin{aligned}
& \text { if }\left(b_{j}=\text { true }\right) \text { then } \\
& o_{j} \cdot l_{j_{1}}=l_{j_{1}} ; o_{j} . l_{j_{2}}=l_{j_{2}} ; \ldots \\
& \text { traverse } f\left(\text { node. } f_{r}, \text { null, } \ldots, o_{j}, \ldots, \text { false }, \ldots, b_{j}, \ldots\right)
\end{aligned}
$$

In other words, if traversal $t_{j}$ is still active by the time it gets to the call, it initializes $o_{j}$ with the necessary parameters to execute traversal $t_{j}$, and passes in null for all the other objects. It then passes $b_{j}$ into the recursive call (signifying that if $t_{j}$ is still active, it should continue to child $f_{r}$ ),

\footnotetext{
${ }^{5}$ We use this level of indirection, rather than directly passing local variables, because not all local variables might exist at a given call site, as we will see later. In such cases, we pass null for the relevant parameter.
} 


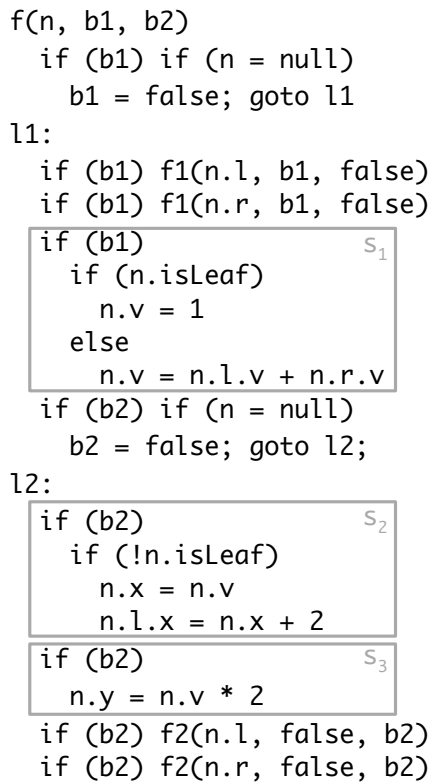

Fig. 4. Single traversal function for running example

and passes false for all the other boolean variables (signifying that, for this call, no other traversal is active).

Running example. Figure 4 shows the result of using this transformation to produce a single traversal covering $\mathrm{f} 1$ and $\mathrm{f} 2$ from our running example. Note that this fused traversal clearly performs exactly the same work as the original traversals-indeed, it generates exactly the same schedule of computation as in Figure 1(c).

\subsection{Generating Code for Fused Calls}

So what happens if the dependence graph we are synthesizing code from contains fused calls? Recall that when a vertex in the dependence graph represents a fused call, its label captures the set of traversals that the call belongs to. Consider generating code for a fused call with label $\left(\left\{t_{i}, t_{j}\right\}, f_{r}\right)$ (a call to child $f_{r}$ from traversals $t_{i}$ and $t_{j}$ ). The generated code for this call is:

$$
\begin{aligned}
& \text { if }\left(b_{i}=\text { true } \vee b_{j}=\text { true }\right) \text { then } \\
& \text { if }\left(b_{i}=\text { true }\right) \text { then } o_{i} \cdot l_{i_{1}}=l_{i_{1}} ; \ldots \text { else } o_{i}=\text { null } \\
& \text { if }\left(b_{j}=\text { true }\right) \text { then } o_{j} . l_{j_{1}}=l_{j_{1}} ; \ldots \text { else } o_{j}=\text { null } \\
& \text { traverse }_{f} \text { (node. } f_{r}, \text { null, } \ldots, o_{i}, o_{j}, \ldots, \text { false }, \ldots, b_{i}, b_{j}, \ldots \text { ) }
\end{aligned}
$$

The fused call occurs when either of the two traversals are active. The call takes in both sets of local variables (if both calls are active) and passes in the active status of both traversals. Hence, if both $t_{i}$ and $t_{j}$ are active when this call is made, the resulting invocation will execute the statements from both $t_{i}$ and $t_{j}$. Note that no other part of the generated code needs to change. All compound statements can keep their same, single-traversal guards.

Applying this rewrite to the fused graph in our running example (Figure 1(e)) yields the code of Figure 1(f). Note that this code has redundant checks and unnecessary checks elided for clarity (for 
example, both null checks occur at the beginning of the method body and return if $\mathrm{n}=$ null, so can be combined into a single, unguarded null check).

\subsection{Integrated Code Motion}

An interesting side effect of the fusion and synthesis procedure is that code motion is naturally and implicitly incorporated. The dependence graph tracks dependences between statements and calls without regard to their original position in their various traversals (indeed, the only time program order matters in the dependence graph is in determining the targets of control dependence edges and determining the direction of dependence edges). As a result, if a statement must move and can move to enable fusion, the fusion procedure will discover this and the synthesis process will implement that code motion through its topological sort. If the necessary code motion cannot happen due to the existence of other dependences, cycles in the graph will make that clear.

In our running example, statement $s_{3}$ is preorder work in traversal f2. However, because of its dependence on $s_{1}$, it must appear after $s_{1}$ in any fused traversal. $s_{1}$, in turn, must appear as post-order work due to its dependences on the calls to the left and right children. The only way to achieve both objectives is to move $s_{3}$ to be post-order work as well, which the topological sort achieves.

\section{SOUNDNESS}

There are two correctness criteria that must be met for fusion to be sound. First, we must preserve work: all of the operations performed by a given traversal in the unfused code must be performed in the fused code, and no more. In other words, each traversal must visit exactly the same portions of the tree it did before, and no more. Second, we must preserve dependences: if two operations have a dependence, then the operations must occur in the same order both in the original, unfused code and in the fused code. We address these two criteria in turn.

\subsection{Preservation of Work}

The first step in proving soundness is confirming that the transformed code performs exactly the same amount of work as the original code-in other words, exactly the same set of operations (compound statements and calls) are performed. We will show this assuming that all the dependences are satisfied (a fact we will prove in the next section); as a result, as long as the fused traversal executes the same set of operations as the original traversals, we can be sure that the operations each behave the same way.

THEOREM 7.1. As long as all dependences are satisfied, a fused traversal method synthesized from the dependence graph will execute exactly the same set of operations as in the original, non-fused traversals.

Proof. We show two things: i) if a statement or call executes in the original code, it will execute in the transformed code; ii) if a traversal truncates in the original code-meaning that traversal stops for a particular subtree-the same will happen in the transformed code.

(1) If a statement executes in the original code, then execution of its traversal must have proceeded to this statement from the root of the tree without encountering a return. In the transformed code, the boolean flag controlling execution of this traversal starts as true. It can only be set to false upon encountering a return. As long as the flag is true, because we are assuming all dependences are satisfied, the traversal will make all of the same calls as the original traversal until it arrives at the statement in question, which will execute.

(2) A traversal truncates in the original code by either executing return, ensuring that it will not visit its children, or by encountering a base case and no longer making child calls. If 
truncation happens due to a return, the transformed code skips over the remainder of the compound statement and sets the boolean flag to false. This ensures that no more work from the traversal will happen at the current node and, because that boolean flag cannot be re-set to true without returning from the current node, ensure that no more work will be performed even if the traversal descends into children from the current node.

Hence, statements in the fused traversal will execute iff they executed in the original code.

\subsection{Preservation of Dependences}

The other aspect of soundness is the preservation of dependences: if two (dynamic) statements occur in a particular order in the original code, and there is a dependence between them, they must execute in the same order in the fused code. To do otherwise would lead to different computational results Note that we do not care about the relative ordering of statements that do not have dependences between them (indeed, the entire point of fusion and other transformations is to alter the execution order of these statements). We also assume here that the trees being traversed are finite: each traversal will eventually terminate.

THEOREM 7.2. Given an original, acyclic dependence graph, $G$, obtained from a set of traversals $T, a$ dependence graph $G^{\prime}$, obtained by fusing two calls in $G$, and the traversal function $t^{\prime}$, synthesized from $G^{\prime}$ : if $G^{\prime}$ is acyclic, then all statements that are ordered by data or control dependences in $T$ execute in the same order in $t^{\prime}$.

Proof. We consider all possible cases of dependences between statements and calls, both within a single traversal in $T$ and across multiple traversals in $T$, and argue that if a dependence exists in $T$, it will be preserved in $t^{\prime}$. We denote the $j$ th statement in traversal $i$ as $s_{i, j}$, and the $j$ th call to the $k$ th child in traversal $i$ as $c_{i, j}^{k}$. All statements and calls are relative to executing at a particular node in the tree, and hence dependences between statements only occur at that particular node. If any dependence occurs between operations at different nodes, that dependence must be between a statements and a call, or between two calls. Note that here we rely on the sound overapproximation of dependences produced by the static analysis for building the dependence graph (Section 4).

$s_{i, j} \rightarrow s_{i^{\prime}, j^{\prime}}, s_{i, j} \rightarrow c_{i^{\prime}, j^{\prime}}^{k}, c_{i, j}^{k} \rightarrow s_{i^{\prime}, j^{\prime}}$ : If $i=i^{\prime}$, there is a dependence between two statements or a statement and a call from the same traversal in the original code and this dependence is captured by $G$. In any fused dependence graph, this edge still exists. A topological sort of the dependence graph will require that the first statement appear before the second in $t^{\prime}$, so the dependence is preserved.

If $i \neq i^{\prime}$, this dependence is between two statements/calls in different traversals. Note that for the dependence to be in this order, traversal $t_{i}$ must have occurred before traversal $t_{i^{\prime}}$. This dependence will be reflected in $G$ and preserved in $G^{\prime}$, and, in $t^{\prime}$, the operation from the first traversal will appear before the operation from the second. Because the dependence occurs when the two traversals are at the same node, the two operations' appearing in the same order in $t^{\prime}$ suffices to preserve the dependence.

$c_{i, j}^{k} \rightarrow c_{i^{\prime}, j^{\prime}}^{k^{\prime}}:$ These are two calls, which are captured as separate vertices in $G$. If $k \neq k^{\prime}$ or if $k=k$ but the two calls are unfused in $G^{\prime}$, then this dependence is preserved by the same argument as the previous case.

When the two calls are fused is the interesting case. In the original code, all of the first call will complete before all of the second call. The dependence exists because the first call executes one or more statements that one or more statements in the second call depend on. We must show that, despite the fusion of the two calls, the necessary statements from the first call still 
execute before the relevant statements from the second. We show this by induction on the maximum depth of the call stacks that originate from the two calls.

We denote the maximum depth of the call chain originating from $c_{i, j}^{k}$ as $k_{i}$, and from $c_{i^{\prime}, j^{\prime}}^{k^{\prime}}$ as $k_{i^{\prime}}$. Note that $k_{i}$ and $k_{i^{\prime}}$ are bounded by the depth of the tree, as each recursive call moves down to a child node of the tree. Without loss of generality, assume that $k_{i}<k_{i^{\prime}}$.

Base case: $k_{i}=1$ : If both $t_{i}$ and $t_{i^{\prime}}$ start at node $n$ and invoke $c_{i, j}^{k}$ and $c_{i^{\prime}, j^{\prime}}^{k^{\prime}}$, respectively, ending at node $n^{\prime}$, there is a dependence between the two traversals at node $n^{\prime}$. Because dependences in the dependence graph are captured relative to an arbitrary node, this dependence at $n^{\prime}$ is captured in the dependence graph $G$. Moreover, because $k_{i}=1$, the dependence must be from a statement from $t_{i}$ to a statement or call in $t_{i^{\prime}}$. Hence, this dependence must also exist in $G^{\prime}$. The topological sort ensures that the (static) statement that leads to the operation in $t_{i}$ will occur before the (static) statement that leads to the operation in $t_{i^{\prime}}$, and hence in the fused case, when both traversals descend to the child at the same time, the dependence will be preserved, by the same argument as in the first case above.

Inductive hypothesis: $k_{i}=l$ : Assume that if the call chains rooted at $c_{i, j}^{k}$ are at most $l$ calls deep, that the dependence is guaranteed to be preserved.

Inductive case: $k_{i}=l+1$ : Consider descending one call deep into the call chain (i.e., both $t_{i}$ and $t_{i^{\prime}}$ make their first recursive calls). There must be at least one dependence between some operation in $t_{i}$ and some operation in $t_{i^{\prime}}$ (including between successive recursive calls). Again, these dependences must be captured by $G$. If the dependences involve any operations except $c_{i, j}^{k}$ and $c_{i^{\prime}, j^{\prime}}^{k^{\prime}}$, then the dependences are also captured by $G^{\prime}$, and by the arguments from above, these dependences are preserved by the fused traversal. If the dependences are between $c_{i, j}^{k}$ and $c_{i^{\prime}, j^{\prime}}^{k^{\prime}}$, then, because we have moved one level down in the tree, the maximum depth of the call chain rooted at $c_{i, j}^{k}$ is now $l$. Appealing to the inductive hypothesis, this dependence is preserved.

Hence, all dependences that exist between traversals in $T$ are overapproximated and captured in $G$, and, if two calls in $G$ are fused to produce $G^{\prime}$, all such dependences are preserved by the traversal synthesized from $G^{\prime}$.

Note that Theorem 7.2 can be applied recursively: the fused graph $G^{\prime}$ is, itself, a valid dependence graph for a recursive traversal. Further fusion that does not create cycles is valid by the same argument.

\section{IMPLEMENTATION}

TreeFuser is implemented as a Clang ${ }^{6}$ compiler tool. It implements the process of generating a dependence graph, identifying candidates for fusion, and synthesizing a new traversal function, as detailed in the previous sections. The implementation is publicly available for download ${ }^{7}$.

\subsection{Implementation Limitations}

Because our prototype implementation of traversal fusion focuses on the challenges specific to this problem, TreeFuser relies on several annotations and restrictions to simplify the implementation:

(1) Rather than building a shape analysis to verify the "treeness" of the structure being traversed [Ghiya and Hendren 1996; Sagiv et al. 1999], TreeFuser relies on annotations to identify tree data structures and recursive fields with in those structures .

\footnotetext{
${ }^{6}$ http://clang.llvm.org

${ }^{7}$ https://bitbucket.org/plcl/treefuserrelease.git
} 
(2) TreeFuser does not allow pointers in the traversal function (except for the operating tree node). However TreeFuser allows the existence of pointers and loops in external "leaf" 8 functions, which use annotations to abstract the access effects of the functions rather than analyzing them. These annotated functions are the only functions allowed to be called in the body of the traversals (other than the recursive traversing function calls).

(3) Section 4.1 mentions some other restrictions of the language that exists in TreeFuser as well (i.e: traversals should have void return types), a more robust implementation can relax many of them as mentioned earlier.

These restrictions are not fundamental limitations of the fusion approach, but instead are limitations of TreeFuser's prototype implementation. Many of these restrictions can be relaxed by using more sophisticated analyses in the access-path construction process (e.g., alias analysis to analyze heap accesses in the program, interprocedural analysis to handle function calls, and widening operators to account for potentially unbounded control flow in traversal bodies). Adding such analyses to the implementation would both reduce the annotation burden and lift many of the restrictions on the language. Nevertheless, the annotation burden is quite low even in our prototype implementation. The next section discusses these annotations in more detail.

\subsection{Annotation Usage}

TreeFuser's prototype implementation uses annotations on data structures and effect annotations on "leaf" functions to avoid the need to implement shape and alias analyses and to allow users to implement more complex control constructs than its input language allows. This section briefly describes these annotations and shows an example of using these annotations in a program. TreeFuser has four types of annotations:

(1) TF_TreeStructure : A class annotation used to identify tree structures.

(2) TF_TreeRecursiveField: A class member annotation used to identify recursive fields within the tree structure. This annotation, and the previous one, are used in lieu of a shape analysis to identify tree data structures.

(3) TF_Traversal : A function annotation that is used to direct TreeFuser to the functions that it should consider for fusion. TreeFuser analyzes and validates the compliance of the those functions with the restrictions imposed by the language, and only valid traversals are considered for fusion.

(4) TF_AbstractAccess ((id, access_type,access_location $) \mid \ldots)$ : An effect annotation on (leaf) functions that summarizes the read and write effects of these functions called from traversal functions. These leaf functions have no restrictions on the operations they perform, provided they are annotated with a TF_AbstractAccess annotation. The effect summaries are given as a list of tuples, each of which consists of:

(a) id: An abstract id that refers to an accessed location or data structure. We use ids to allow treating operations on complex data types (e.g., inserting a key into a set) as a single read or write on an abstract location.

(b) access_type: Whether the access is a read ('r') or write ('w').

(c) access_location: Indicates whether the accessed location is a local to the current node of the tree or global and accessible from all nodes. Note that if the leaf function function accesses a local abstract location then it must be a class member.

TreeFuser looks for sequences of "potentially fusable" methods in the remainder of the code that operate on the same tree and attempts to perform fusion as outlined in Section 5.

\footnotetext{
${ }^{8}$ We call these functions leaf functions because they do not call back to any of the traversal functions considered for fusion.
} 


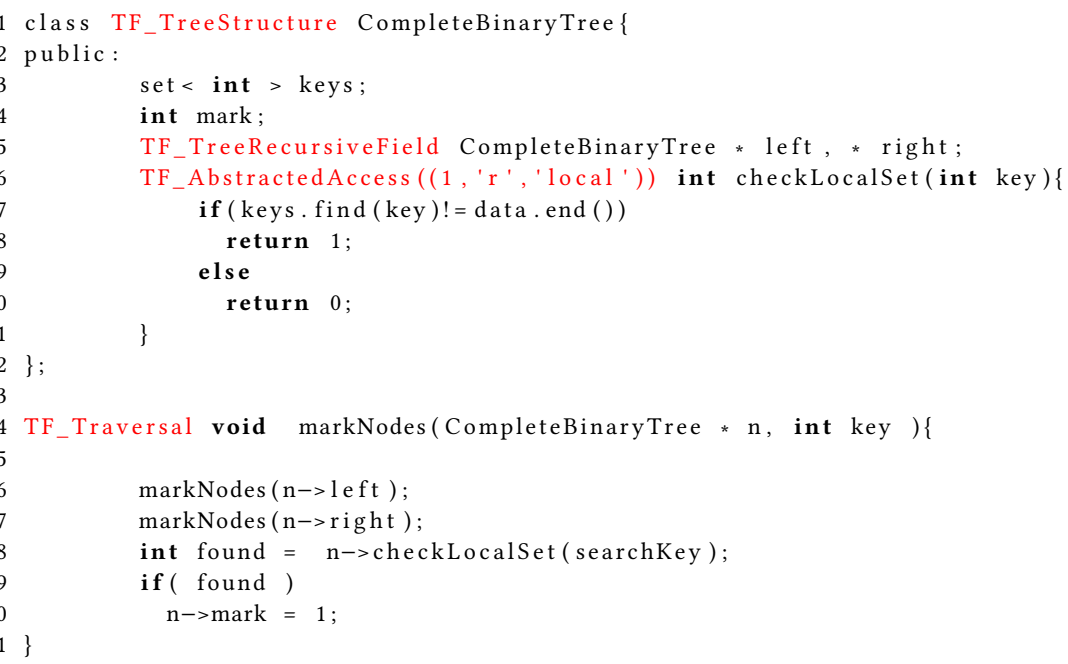

Fig. 5. TreeFuser Example

Figure 5 shows an example of a simple program written in TreeFuser that uses the different annotations described earlier: a definition of binary tree with a set of integers stored at each node, keys, along with a mark flag. markNodes is a traversal that operates on the tree, marking nodes that have the given input key stored in its keys set. An annotated function, checkLocalset is used to do the local search in the keys set, the set keys is abstracted by the id 1 and any functions that access keys should use the same id.

\subsection{Fusion Heuristic}

TreeFuser's dependence graph abstraction prescribes a test for when call fusion is legal, and its synthesis procedure generates valid fused traversal methods for any legal dependence graph. However, the particular order in which calls are fused is open to exploration. Unfortunately, the space of possible fused traversals is vast. Choosing to fuse together children in different orders can introduce different sets of dependences (because of the vertex merging step in the fusion process), and hence different choices for fusion can lead to different performance characteristics.

TreeFuser implements a simple greedy heuristic. It chooses a call vertex in the graph as the target call. It then iterates through each remaining unfused call and attempts to fuse it with the target call. If the call cannot be fused with the target it is set aside. Once all unfused calls have been attempted to be fused with the target, TreeFuser chooses another unfused call as the new target call. This process repeats until no more fusion is possible.

Ultimately, what we desire is a maximization of dynamic fusion: having as many traversals overlap as much as possible during the execution of the traversal method(s) on a particular tree. Sadly, heuristics such as maximizing the number of static calls that are fused (already a challenging objective, due to the combinatorial search space) may not achieve this goal: fusing together several calls may leave the two calls that account for the most work in a particular tree unfused. Dynamic fusion is a run-time property and hence is not particularly amenable to static optimization, though profiling information may help. We leave a full exploration of effective fusion heuristics to future work. However, our evaluation does look at the potential effect of the fusion heuristic on the performance of the fused schedule (Section 9.4). 


\section{EXPERIMENTS AND EVALUATION}

As discussed in the introduction, there are many applications of traversal fusion: improving locality or parallelism, eliminating intermediate structures, increasing optimization opportunities, etc. The common factor uniting these applications is reducing the number of node accesses by the set of traversals. We evaluate three case studies, demonstrating that we are able to substantially reduce the number of times tree nodes are visited and enhance the performance and the locality of the traversals:

(1) We fuse two passes from the popular Fast Multipole Method (FMM) [Greengard and Rokhlin 1987].

(2) We fuse multiple DOM traversals in a document rendering engine, written in a general language.

(3) We fuse 6 interlocking abstract syntax tree passes in a small compiler, each written as a fine-grained traversal ${ }^{9}$ in a general language.

All three case studies require TreeFuser's code motion to perform effective fusion. While the FMM case study's passes can be totally fused, the other two case studies also require TreeFuser's partial fusion to obtain any benefits. We also evlaute the sensitivity of the fusion transformation to the fusion heuristic by comparing the performance of different fusing schedules of the AST passes

Experimental platform. As mentioned in Section 8, TreeFuser is written as a Clang tool. Traversals are written in $\mathrm{C}++$, and annotated as described in Section 8 for processing with TreeFuser. After processing, the synthesized code is compiled with LLVM version 3.8.0. The execution platform for the various performance runs is a dual 12-core, Intel Xeon $2.7 \mathrm{GHz}$ Core with $32 \mathrm{~KB}$ of L1 cache, $256 \mathrm{~KB}$ of L2 cache, and $20 \mathrm{MB}$ of L3 cache.

\subsection{Case Study 1: Fast Multipole Method}

The fast multipole method (FMM) is a numerical technique used to evaluate all pairwise interactions of a large number of points distributed in a space (e.g. long-ranged forces in the n-body problem, gravitational potential, and computational electromagnetic problems) [Engheta et al. 1992; Rokhlin 1985].

In this case we are considering the application of computing the gravitational potential for a large set of points distributed in a two dimensional space, the points are arranged in quad-tree where each leaf node contains a subset of the points that reside within a specific subspace, and interior nodes have summary information (e.g., the center of mass of all descendant points).

FMM is typically implemented as multiple top-down and bottom-up passes over the quad-tree. We consider two of these passes: the first traversal, which updates the potential of each node based on its siblings, and the second traversal, which propagates these values down the tree. We made slight modifications to the FMM implementation from the Treelogy benchmark suite [Hegde et al. 2017] to fit the code into our restricted language (these modifications mostly consisted of moving complex mathematical computations into functions annotated with-obvious-read and write effects).

TreeFuser was able to fully fuse the two passes and exhibit a performance improvement up to $35 \%$ over the unfused version, Figure 6 shows the speedups for different input sizes.

\subsection{Case Study 2: Fusing Render-tree Passes}

Tree traversals are common in any document rendering engine, including in web browsers. Considering a simple scenario: once the web page is acquired as DOM (Document Object Model) and

${ }^{9}$ These traversals are too complex to easily express using attribute grammars. 


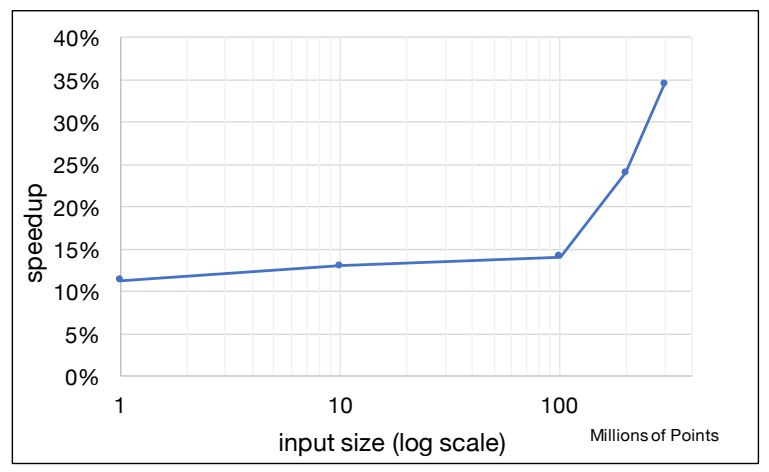

Fig. 6. Runtime speedups of the fused traversals for different number of points.

Table 1. Performance of fused render-tree traversals

\begin{tabular}{|l|l|}
\hline Performance Parameter & Value \\
\hline Runtime Speedup & 1.4515 \\
Normalized L2 Data Cache Accesses & 0.5688 \\
Normalized L2 Data Cache Misses & 0.5473 \\
Normalized L3 Cache Accesses & 0.5474 \\
Normalized L3 Cache Misses & 0.4843 \\
\hline
\end{tabular}

CSSOM (CSS Object Model), the browser rendering engine constructs what is known as a rendertree. Then the rendering engine performs multiple traversals upon this tree to display the content on screen: determining the position of objects on the screen, the style of elements, etc. While these traversals individually appear to be simple, in the presence of multiple traversals it is not trivial to fuse them manually. Automatically fusing these traversals can enhance the performance of the rendering engine without sacrificing programmability.

We have implemented four different traversals sequentially performed on a binary render-tree. ${ }^{10}$ These traversals closely follow the ones shown in Meyerovich et al. [2013]. These traversals require partial fusion: all four traversals can be fused while visiting the left child, visits to the right child are decomposed into sets of two traversals. When rendering a tree with $2^{19}-1$ elements, the unfused traversals visit 1835004 nodes in total, while the fused traversals visit 1048555 nodes, a reduction in node visits of $42.9 \%$. Table 1 illustrates the performance benefits of fusing render-tree traversals: TreeFuser delivers a $45 \%$ performance improvement, driven by substantial reductions in L2 and L3 cache accesses and misses.

\subsection{Case Study 3: Fusing AST Passes}

We implemented a compiler for a simple language (essentially, typed IMP) that builds an abstract syntax tree (AST) and then performs several AST passes over that tree to perform various compilation steps. The AST, which is naturally written using several different node types, and the traversals are rewritten according to the procedure described in Section 4.1 to yield a single tree node type. The resulting tree nodes have 12 different possible children. The six AST passes vary in complexity from performing constant folding, to a pair of passes that together perform available expressions analysis (avoiding the iterative nature of dataflow analysis by taking advantage of the structured nature of the AST [Farrow et al. 1976]). Table 2 lists the six passes, their size in lines of code, and

\footnotetext{
${ }^{10}$ Render-trees are typically binary trees: larger out degrees are captured with the equivalent of "head" and "rest" pointers.
} 
Table 2. Details of AST passes

\begin{tabular}{|l|r|r|r|}
\hline Pass & LoC & \# of calls & $\begin{array}{r}\text { Cumul. analysis } \\
\text { time (ms) }\end{array}$ \\
\hline Constant folding & 50 & 12 & 123 \\
Constant prop. & 96 & 13 & 137 \\
Find uses & 40 & 12 & 160 \\
Find defs & 47 & 7 & 176 \\
Computed exprs. & 63 & 10 & 187 \\
Available exprs. & 100 & 12 & 225 \\
\hline
\end{tabular}

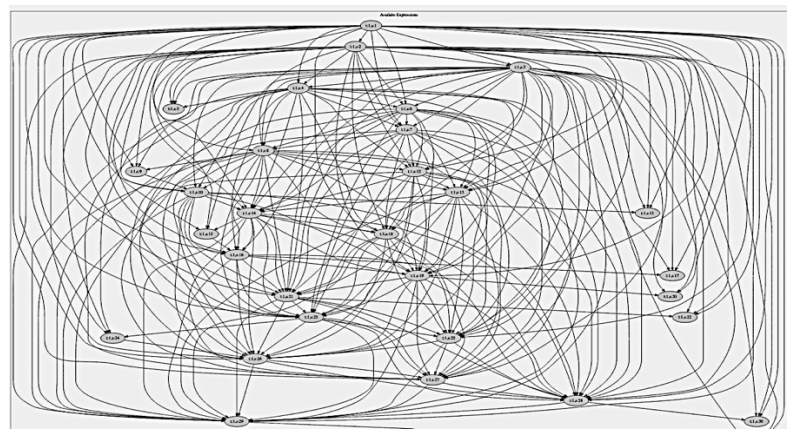

Fig. 7. Dependence graph for available expressions pass.

the number of recursive calls they make. These passes are called in succession. Notably, the passes have complex dependences on one another. For example, constant folding changes the expressions in the AST, which affects which expressions are available. To get a sense of how complex the traversals are, Figure 7 shows the dependence graph for just the available expressions pass-manual fusion of these passes would be impractical. Further, these passes are non-trivial to express using formalisms such as attribute grammars due to their complexity (e.g., constant propagation visits one child type twice, which would have to be decomposed into multiple attributes for expression in an attribute grammar).

TreeFuser performance. The final column in Table 2 shows the amount of time TreeFuser takes to analyze the dependences in a set of passes and synthesize a fused traversal. Because analysis time depends on the number and complexity of the passes analyzed, we give times for various configurations of passes. An entry in the column for a given analysis pass gives the analysis time for a configuration containing all of the passes in the table from the first row to the row in question (e.g., the entry for the constant prop. pass gives the time to fuse the constant prop. and the constant folding passes, while the entry for the last row gives the time to fuse a program that uses all six passes).

As we can see, TreeFuser takes very little time to analyze and generate fused traversals. Note, moreover, that most of the analysis time goes in fixed overhead (e.g., analyzing the code to find fusable traversals). The analysis complexity itself is $O\left(n^{2}\right)$ where $n$ is the number of statements. (Generating the access paths is $O(n)$, as our target language does not contain loops, and building the dependence graph is $O\left(n^{2}\right)$ as all pairs of statements/calls must be compared. The complexity of performing fusion could, in theory, be higher depending on the complexity of the fusion heuristic used.) In particular, going from analyzing 50 lines of code (row 1) to 400 (row 6) adds only about.1 seconds to the total analysis time. 


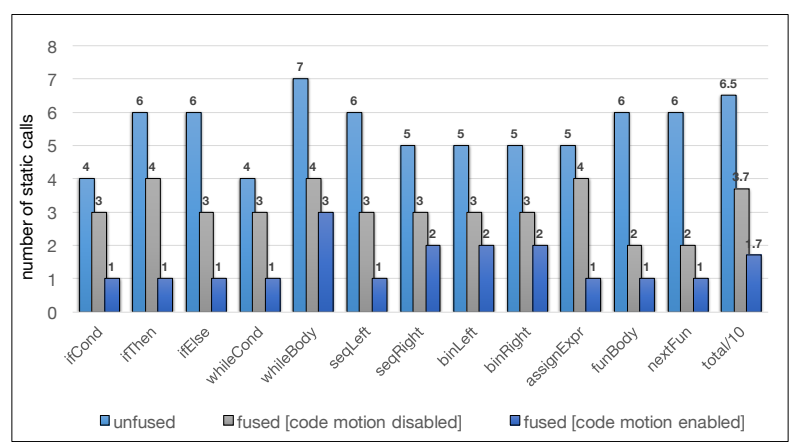

Fig. 8. Comparison between the number of static recursive calls on each child, and total, across all traversals ("total" bar is scaled by a factor of 10 to fit on the chart).

Static fusion effectiveness. The first measure of effectiveness we study is static fusion effectiveness: given the six passes, each of which invokes some number of child calls, how many of those calls is TreeFuser able to fuse together? Figure 8 shows the number of calls made to each recursive field type in the tree node ${ }^{11}$. We find three things: first, some traversals cannot be fully fused with other traversals, however fusing some calls in those traversals is possible, which indicates that partial fusion is necessary to achieve better reduction in traversal calls. Second, fusion with code motion turned off produces a fused traversal with 37 calls (versus 65 calls in the baseline traversals). Finally, enabling code motion fuses the traversal further to just 17 calls. These results suggest that the two key novelties of TreeFuser's transformations, generalized code motion and partial fusion, are necessary to perform effective fusion.

Dynamic fusion effectiveness. Looking at static call fusion alone does not tell the entire story. Two calls may be fused, but be for portions of the AST that do not account for much of the traversal. Instead, we also want to investigate dynamic fusion effectiveness: for a test suite of programs, how many traversal calls are eliminated by fusion. We examine three different input programs of different sizes; Figure 9 shows the number of times the AST nodes are visited by the different configurations of traversals: unfused, fused without code motion, and fused with code motion. We can see that without code motion, we can reduce the number of node visits by $19 \%$ in aggregate. When combined with code motion, we are able to reduce AST node visits by $56 \%$ in aggregate, demonstrating the ability of TreeFuser to substantially coarsen the computation at each node in the tree.

Performance. Fusion has the potential to enhance locality by bringing accesses to same set of data closer. However, the specific improvements in locality depend on the amount of data reuse between traversals, and the overall footprint of the computation. Figure 10(a) shows the reduction in L2 and L3 cache misses for three different input programs. The amount of reduction in the cache misses differs from one program to another, but in all cases there is a substantial decrease in the number of misses in both levels of cache.

The reduction in the cache misses directly affects the runtime of the traversals, Figure 10(a) shows the runtime speedups for the the three programs, with different sizes (larger sizes are generated by replicating the functions in the base program with different names, to simulate larger whole-program compilation of programs with similar AST structures). For small ASTs, the runtime

\footnotetext{
${ }^{11}$ Note that whileBody has seven calls, even though there are only six passes; this is because the constant propagation AST pass visits the body of the while loop twice.
} 


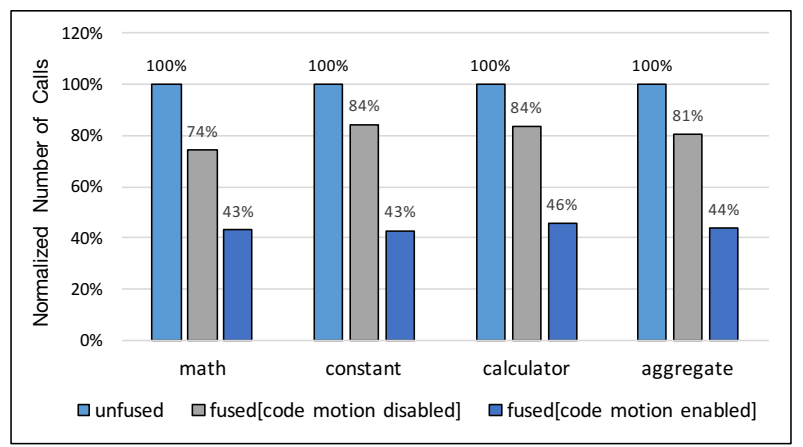

Fig. 9. Dynamic number of node visits across three programs for unfused traversals, fused without code motion, and fused with code motion. The numbers of visits is normalized to the number of visits in the unfused traversals.

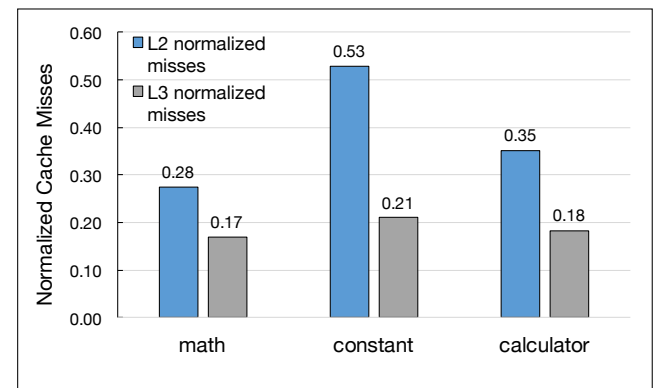

(a) Cache misses for the fused version normalized to the unfused version.

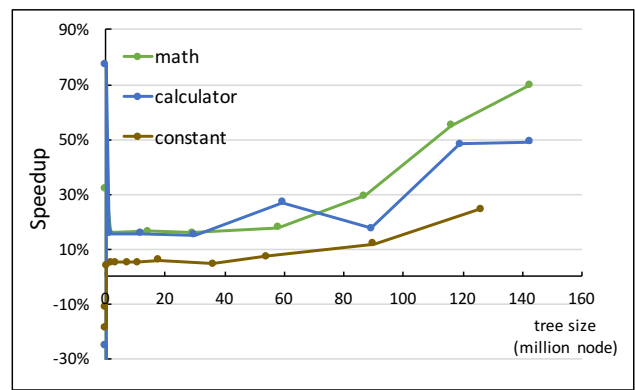

(b) Performance of fused traversals over unfused traversals for three AST structures

Fig. 10. Cache and Runtime Statistics

is very short, and subject to measurement noise, hence there is no consistent performance story. ${ }^{12}$ Once the ASTs grow large enough to fill the cache, performance improvements can be seen, with speedups of up to $70 \%$.

Note that ASTs are not data-intensive structures and the interaction between the accesses in most traversals is limited to some fields and the node pointer. We would expect a larger performance improvement in data intensive applications with larger chunks of data reuse. AST traversals were chosen here to show the ability of TreeFuser to fuse complicated traversals.

Overhead. There are two sources of overhead for the fusion transformation. First is an increase in instruction overhead, due to the additional guards inserted by (partial) fusion. The measured instruction overhead for the AST passes was relatively low: 10\% or less for all three input programs. A second source of overhead is an increase in instruction misses due to the larger code footprint of fused code. While this overhead is noticeable for small inputs, for larger inputs the large decrease in data cache accesses and misses more than compensates.

\footnotetext{
${ }^{12}$ Note that performing fusion for small trees is akin to performing loop tiling for small matrices-there is no opportunity for performance gains since the whole structure fits in cache.
} 


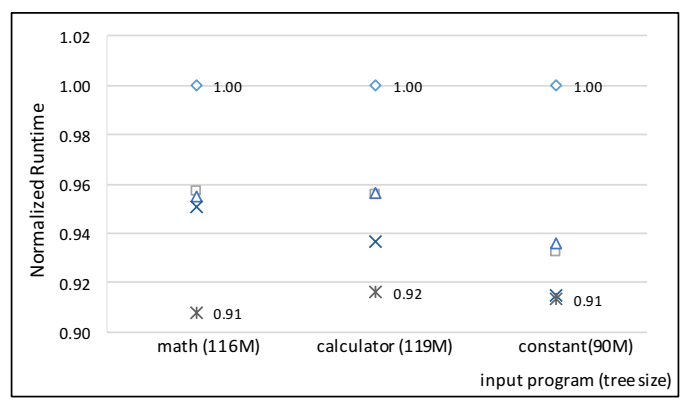

Fig. 11. Sensitivity analysis of fusing and ordering criteria. The $X$ axis shows the input name and the number of nodes for each of the inputs; the $\mathrm{Y}$ axis shows the runtime of the different schedules normalized to the best schedule.

\subsection{Sensitivity Analysis}

Different fused schedules might be generated using the general approach discussed in this paper: the fusion heuristic used in constructing the fused graph (Section 8.3), along with the chosen topological order (Section 6.1), determine the final fused schedule. TreeFuser uses a greedy approach for generating the fused graph and an arbitrary topological order in the synthesis process. This section studies the sensitivity of performance to these choices.

Five different fused schedules of the AST passes are studied. The fused graphs of these schedules were generated by applying the greedy approach that is used in TreeFuser, but with the nodes being merged in a different random order each time (exploring more sophisticated fusing heuristics is beyond the scope of this paper).

Figure 11 shows the runtime of these five fused schedules, normalized to the best schedule among all of them. It is worth mentioning first that even the worst-performing schedule shows a significant speedup of $(34 \%, 26 \%$ and $6 \%)$ for the three input programs respectively from left to right. While the performance differences are statistically significant, there is only about a $10 \%$ difference between the best-performing and worst-performing fusion choices. The results shows that it will be useful to further explore the space of fusion policies, yet, even with an arbitrary choice, fusion gives a decent speedup.

\section{FUTURE WORK}

There are many opportunities to extend the analyses and implementation of TreeFuser. As discussed in Section 8, the implementation of TreeFuser can be made more robust, allowing many of the limitations of the specification language presented in Section 4.1 to be relaxed. These implementation extensions mostly concern making the static analyses that identify dependences between statements more powerful by incorporating known techniques such as interprocedural shape analyses. However, there are other avenues of future work that require extending the framework in novel ways.

As discussed in Section 4.1, one key limitation of the current framework is that it assumes that all nodes in the tree are of the same type. Heterogeneous trees (such as ASTs) are essentially handled by overapproximating the operations that can be performed on a given tree node, assuming the node could be any possible type. Unsurprisingly, this results in conservative analysis results: dependences could arise due to statements whose execution would require impossible combinations of node types. Extending the identification of dependences between statements to be type aware, both based on global constraints (e.g., the right child of an expression node in an AST can never be 
an if statement) and local, conditional constraints (e.g., a particular piece of code only executes if a expression node's child is a literal) could allow us to eliminate spurious dependences and expose more opportunity for fusion.

As discussed in Section 8.3, TreeFuser currently implements a simple, greedy strategy for determining which recursive calls to fuse. As a result, there is a potentially wide variation in the performance of the fused code, depending on which fusion choices are made (as demonstrated in Section 9.4). There is substantial work to be done in developing good strategies for fusion, such as profile-driven fusion (fusing calls that are more likely to have reuse) or auto-tuning.

A final area to explore for future work concerns applying fusion to traversal applications that do not fit the recursive template we currently support. For example, code that expects to traverse large trees may adopt explicit stack-based implementations rather than recursive implementations to avoid stack overflow. Determining both what "fusion" means in this context, as well as how to identify and exploit opportunities for fusion, is an interesting direction.

\section{CONCLUSIONS}

This paper presented TreeFuser, a framework for performing fusion of general tree traversals written in an imperative language. Unlike previous work on fusion of tree traversals, TreeFuser allows programmers to write a series of small, fine-grained tree traversals in a general-purpose language, without the use of ad hoc, high-level abstractions, and then automatically merges the traversals together to perform coarser-grained operations at each node in the tree. TreeFuser integrates two key novelties: general code motion that allows more operations to be fused together, and partial traversal, that allows for the coarsening of computation even if two traversals are not fully fusable.

The enabling techniques for TreeFuser's transformations are a dependence representation that can be extracted from general traversal code, a dependence test that allows the framework to determine the validity of fusion while accounting for code motion, and a synthesis procedure that allows complex traversals to be synthesized from this high level abstraction. We show through three case studies that i) when there is reuse between traversals, TreeFuser is able to provide good performance benefits; and ii) TreeFuser is able to perform intricate partial fusion and code motion on a series of styling passes over a render-tree and AST traversals in a compiler, dramatically reducing the number of node accesses in both cases.

\section{ACKNOWLEDGMENTS}

The authors would like to thank Nikhil Hegde for providing the FMM implementation used in Case Study 1 . We would also like to thank Leo Meyerovich for sharing his document-rendering code. Finally, the authors would like to thank the anonymous referees for their helpful feedback. This work was supported in part by the following grants: NSF CCF-1150013, NSF CCF-1439126, and DOE DE-SC0010295.

\section{REFERENCES}

Pierre Amiranoff, Albert Cohen, and Paul Feautrier. 2006. Beyond Iteration Vectors: Instancewise Relational Abstract Domains. In Proceedings of the 13th International Conference on Static Analysis (SAS'06). Springer-Verlag, Berlin, Heidelberg, 161-180. DOI : http://dx.doi.org/10.1007/11823230_11

Uday Bondhugula, Albert Hartono, J. Ramanujam, and P. Sadayappan. 2008. A Practical Automatic Polyhedral Parallelizer and Locality Optimizer. In Proceedings of the 29th ACM SIGPLAN Conference on Programming Language Design and Implementation (PLDI '08). ACM, New York, NY, USA, 101-113. DOI : http://dx.doi.org/10.1145/1375581.1375595

Wei-Ngan Chin. 1992. Safe Fusion of Functional Expressions. In Proceedings of the 1992 ACM Conference on LISP and Functional Programming (LFP '92). ACM, New York, NY, USA, 11-20. DOI : http://dx.doi.org/10.1145/141471.141494 
Albert Cohen and Jean-François Collard. 1998. Instance-Wise Reaching Definition Analysis for Recursive Programs Using Context-Free Transductions. In Proceedings of the 1998 International Conference on Parallel Architectures and Compilation Techniques (PACT '98). IEEE Computer Society, Washington, DC, USA, 332-. http://dl.acm.org/citation.cfm?id=522344. 825716

H. Comon, M. Dauchet, R. Gilleron, C. Löding, F. Jacquemard, D. Lugiez, S. Tison, and M. Tommasi. 2007. Tree Automata Techniques and Applications. Available on: http://www.grappa.univ-lille3.fr/tata. (2007). release October, 12 th 2007.

Loris D’Antoni, Margus Veanes, Benjamin Livshits, and David Molnar. 2014. Fast: A Transducer-based Language for Tree Manipulation. In Proceedings of the 35th ACM SIGPLAN Conference on Programming Language Design and Implementation (PLDI '14). ACM, New York, NY, USA, 384-394. DOI : http://dx.doi.org/10.1145/2594291.2594309

Alain Darte. 1999. On the Complexity of Loop Fusion. In Proceedings of the 1999 International Conference on Parallel Architectures and Compilation Techniques (PACT '99). IEEE Computer Society, Washington, DC, USA, 149-. http: //dl.acm.org/citation.cfm?id=520793.825721

John Doner. 1970. Tree Acceptors and Some of Their Applications. F. Comput. Syst. Sci. 4, 5 (Oct. 1970), 406-451. DOI: http://dx.doi.org/10.1016/S0022-0000(70)80041-1

Torbjörn Ekman and Görel Hedin. 2007. The Jastadd Extensible Java Compiler. In Proceedings of the 22Nd Annual ACM SIGPLAN Conference on Object-oriented Programming Systems and Applications (OOPSLA '07). ACM, New York, NY, USA, 1-18. DOI : http://dx.doi.org/10.1145/1297027.1297029

Joost Engelfriet. 1975. Bottom-up and top-down tree transformations- a comparison. Mathematical systems theory 9, 2 (01 Jun 1975), 198-231. DOI: http://dx.doi.org/10.1007/BF01704020

Joost Engelfriet and Sebastian Maneth. 2002. Output String Languages of Compositions of Deterministic Macro Tree Transducers. F. Comput. Syst. Sci. 64, 2 (March 2002), 350-395. DOI : http://dx.doi.org/10.1006/jcss.2001.1816

Joost Engelfriet and Heiko Vogler. 1985. Macro tree transducers. F. Comput. System Sci. 31, 1 (1985), 71 - 146. DOI http://dx.doi.org/10.1016/0022-0000(85)90066-2

N. Engheta, W. D. Murphy, V. Rokhlin, and M. S. Vassiliou. 1992. The fast multipole method (FMM) for electromagnetic scattering problems. IEEE Transactions on Antennas and Propagation 40, 6 (Jun 1992), 634-641. DOI : http://dx.doi.org/10. $1109 / 8.144597$

R. Farrow, K. Kennedy, and L. Zucconi. 1976. Graph Grammars and Global Program Data Flow Analysis. In Proceedings of the 17th Annual Symposium on Foundations of Computer Science (SFCS '76). IEEE Computer Society, Washington, DC, USA, 42-56. DOI : http://dx.doi.org/10.1109/SFCS.1976.17

Paul Feautrier. 1992a. Some Efficient Solutions to the Affine Scheduling Problem: I. One-dimensional Time. Int. F. Parallel Program. 21, 5 (Oct. 1992), 313-348. DOI : http://dx.doi.org/10.1007/BF01407835

Paul Feautrier. 1992b. Some efficient solutions to the affine scheduling problem. Part II. Multidimensional time. International fournal of Parallel Programming 21, 6 (01 Dec 1992), 389-420. DOI : http://dx.doi.org/10.1007/BF01379404

Paul Feautrier. 1998. A parallelization framework for recursive tree programs. In Euro-Par'98 Parallel Processing: 4th International Euro-Par Conference Southampton, UK, September 1-4, 1998 Proceedings, David Pritchard and Jeff Reeve (Eds.). Springer Berlin Heidelberg, Berlin, Heidelberg, 470-479. DOI : http://dx.doi.org/10.1007/BFb0057890

Rakesh Ghiya and Laurie J. Hendren. 1996. Is It a Tree, a DAG, or a Cyclic Graph? A Shape Analysis for Heap-directed Pointers in C. In Proceedings of the 23rd ACM SIGPLAN-SIGACT Symposium on Principles of Programming Languages (POPL '96). ACM, New York, NY, USA, 1-15. DOI : http://dx.doi.org/10.1145/237721.237724

Rakesh Ghiya, Laurie J. Hendren, and Yingchun Zhu. 1998. Detecting Parallelism in C Programs with Recursive Darta Structures. In Proceedings of the 7th International Conference on Compiler Construction (CC '98). Springer-Verlag, London, UK, UK, 159-173. http://dl.acm.org/citation.cfm?id=647474.727598

Andrew Gill, John Launchbury, and Simon L. Peyton Jones. 1993. A Short Cut to Deforestation. In Proceedings of the Conference on Functional Programming Languages and Computer Architecture (FPCA '93). ACM, New York, NY, USA, 223-232. DOI : http://dx.doi.org/10.1145/165180.165214

L. Greengard and V. Rokhlin. 1987. A Fast Algorithm for Particle Simulations. F. Comput. Phys. 73, 2 (Dec. 1987), 325-348 DOI : http://dx.doi.org/10.1016/0021-9991(87)90140-9

Robert J. Harrison, George I. Fann, Takeshi Yanai, Zhengting Gan, and Gregory Beylkin. 2004. Multiresolution quantum chemistry: Basic theory and initial applications. The fournal of Chemical Physics 121, 23 (2004), 11587-11598. DOI: http://dx.doi.org/10.1063/1.1791051 arXiv:http://dx.doi.org/10.1063/1.1791051

N. Hegde, J. Liu, K. Sundararajah, and M. Kulkarni. 2017. Treelogy: A benchmark suite for tree traversals. In 2017 IEEE International Symposium on Performance Analysis of Systems and Software (ISPASS). 227-238. DOI : http://dx.doi.org/10. 1109/ISPASS.2017.7975294

Ralf Hinze, Thomas Harper, and Daniel W. H. James. 2011. Theory and Practice of Fusion. In Proceedings of the 22Nd International Conference on Implementation and Application of Functional Languages (IFL'10). Springer-Verlag, Berlin, Heidelberg, 19-37. http://dl.acm.org/citation.cfm?id=2050135.2050137

Proc. ACM Program. Lang., Vol. 1, No. OOPSLA, Article 76. Publication date: October 2017. 
Joseph Hummel, Laurie J. Hendren, and Alexandru Nicolau. 1994. A General Data Dependence Test for Dynamic, Pointerbased Data Structures. In Proceedings of the ACM SIGPLAN 1994 Conference on Programming Language Design and Implementation (PLDI '94). ACM, New York, NY, USA, 218-229. DOI : http://dx.doi.org/10.1145/178243.178262

Ken Kennedy and Kathryn S. McKinley. 1994. Maximizing Loop Parallelism and Improving Data Locality via Loop Fusion and Distribution. In Proceedings of the 6th International Workshop on Languages and Compilers for Parallel Computing. Springer-Verlag, London, UK, UK, 301-320. http://dl.acm.org/citation.cfm?id=645671.665526

Donald E. Knuth. 1968. Semantics of context-free languages. Mathematical systems theory 2, 2 (01 Jun 1968), 127-145. DOI : http://dx.doi.org/10.1007/BF01692511

J. R. Larus and P. N. Hilfinger. 1988. Detecting Conflicts Between Structure Accesses. In Proceedings of the ACM SIGPLAN 1988 Conference on Programming Language Design and Implementation (PLDI '88). ACM, New York, NY, USA, 24-31. DOI : http://dx.doi.org/10.1145/53990.53993

Parthasarathy Madhusudan, Xiaokang Qiu, and Andrei Stefanescu. 2012. Recursive Proofs for Inductive Tree Data-structures. In Proceedings of the 39th Annual ACM SIGPLAN-SIGACT Symposium on Principles of Programming Languages (POPL '12). ACM, New York, NY, USA, 123-136. DOI : http://dx.doi.org/10.1145/2103656.2103673

Andreas Maletti. 2008. Compositions of Extended Top-down Tree Transducers. Inf. Comput. 206, 9-10 (Sept. 2008), $1187-1196$ DOI : http://dx.doi.org/10.1016/j.ic.2008.03.019

MÃČ Âşnica MartÃČÂynez and Alberto Pardo. 2013. A shortcut fusion approach to accumulations. Science of Computer Programming 78, 8 (2013), 1121 - 1136. DOI: http://dx.doi.org/10.1016/j.scico.2012.09.002 Special section on software evolution, adaptability, and maintenance \& Special section on the Brazilian Symposium on Programming Languages.

R. L. Mattson, J. Gecsei, D. R. Slutz, and I. L. Traiger. 1970. Evaluation Techniques for Storage Hierarchies. IBM Syst. f. 9,2 (June 1970), 78-117. DOI : http://dx.doi.org/10.1147/sj.92.0078

Erik Meijer and Johan Jeuring. 1995. Merging Monads and Folds for Functional Programming. In Advanced Functional Programming, First International Spring School on Advanced Functional Programming Techniques-Tutorial Text. SpringerVerlag, London, UK, UK, 228-266. http://dl.acm.org/citation.cfm?id=647698.734152

Leo A. Meyerovich and Rastislav Bodik. 2010. Fast and Parallel Webpage Layout. In Proceedings of the 19th International Conference on World Wide Web (WWW'10). ACM, New York, NY, USA, 711-720. DOI : http://dx.doi.org/10.1145/1772690. 1772763

Leo A. Meyerovich, Matthew E. Torok, Eric Atkinson, and Rastislav Bodik. 2013. Parallel Schedule Synthesis for Attribute Grammars. In Proceedings of the 18th ACM SIGPLAN Symposium on Principles and Practice of Parallel Programming (PPoPP '13). ACM, New York, NY, USA, 187-196. DOI : http://dx.doi.org/10.1145/2442516.2442535

Dmitry Petrashko, Ondřej Lhoták, and Martin Odersky. 2017. Miniphases: Compilation Using Modular and Efficient Tree Transformations. In Proceedings of the 38th ACM SIGPLAN Conference on Programming Language Design and Implementation (PLDI 2017). ACM, New York, NY, USA, 201-216. DOI : http://dx.doi.org/10.1145/3062341.3062346

Apan Qasem and Ken Kennedy. 2006. Profitable Loop Fusion and Tiling Using Model-driven Empirical Search. In Proceedings of the 20th Annual International Conference on Supercomputing (ICS '06). ACM, New York, NY, USA, 249-258. DOI: http://dx.doi.org/10.1145/1183401.1183437

Samyam Rajbhandari, Jinsung Kim, Sriram Krishnamoorthy, Louis-Noel Pouchet, Fabrice Rastello, Robert J. Harrison, and P. Sadayappan. 2016a. A Domain-specific Compiler for a Parallel Multiresolution Adaptive Numerical Simulation Environment. In Proceedings of the International Conference for High Performance Computing, Networking, Storage and Analysis (SC '16). IEEE Press, Piscataway, NJ, USA, Article 40, 12 pages. http://dl.acm.org/citation.cfm?id=3014904.3014958

Samyam Rajbhandari, Jinsung Kim, Sriram Krishnamoorthy, Louis-Noël Pouchet, Fabrice Rastello, Robert J. Harrison, and P. Sadayappan. 2016b. On Fusing Recursive Traversals of K-d Trees. In Proceedings of the 25th International Conference on Compiler Construction (CC 2016). ACM, New York, NY, USA, 152-162. DOI : http://dx.doi.org/10.1145/2892208.2892228

V Rokhlin. 1985. Rapid solution of integral equations of classical potential theory. F. Comput. Phys. 60, 2 (1985), 187 - 207. DOI : http://dx.doi.org/10.1016/0021-9991(85)90002-6

Tiark Rompf, Arvind K. Sujeeth, Nada Amin, Kevin J. Brown, Vojin Jovanovic, HyoukJoong Lee, Manohar Jonnalagedda, Kunle Olukotun, and Martin Odersky. 2013. Optimizing Data Structures in High-level Programs: New Directions for Extensible Compilers Based on Staging. In Proceedings of the 40th Annual ACM SIGPLAN-SIGACT Symposium on Principles of Programming Languages (POPL '13). ACM, New York, NY, USA, 497-510. DOI : http://dx.doi.org/10.1145/2429069.2429128

Radu Rugina and Martin C. Rinard. 2005. Symbolic Bounds Analysis of Pointers, Array Indices, and Accessed Memory Regions. ACM Trans. Program. Lang. Syst. 27, 2 (March 2005), 185-235. DOI : http://dx.doi.org/10.1145/1057387.1057388

Mooly Sagiv, Thomas Reps, and Reinhard Wilhelm. 1999. Parametric Shape Analysis via 3-valued Logic. In Proceedings of the 26th ACM SIGPLAN-SIGACT Symposium on Principles of Programming Languages (POPL '99). ACM, New York, NY, USA, 105-118. DOI : http://dx.doi.org/10.1145/292540.292552

Philip Wadler. 1988. Deforestation: Transforming Programs to Eliminate Trees. In Proceedings of the Second European Symposium on Programming. North-Holland Publishing Co., Amsterdam, The Netherlands, The Netherlands, 231-248. http://dl.acm.org/citation.cfm?id=80098.80104 
Yusheng Weijiang, Shruthi Balakrishna, Jianqiao Liu, and Milind Kulkarni. 2015. Tree Dependence Analysis. In Proceedings of the 36th ACM SIGPLAN Conference on Programming Language Design and Implementation (PLDI '15). ACM, New York, NY, USA, 314-325. DOI : http://dx.doi.org/10.1145/2737924.2737972

Ben Wiedermann and William R. Cook. 2007. Extracting Queries by Static Analysis of Transparent Persistence. In Proceedings of the 34th Annual ACM SIGPLAN-SIGACT Symposium on Principles of Programming Languages (POPL '07). ACM, New York, NY, USA, 199-210. DOI : http://dx.doi.org/10.1145/1190216.1190248 